\title{
The Type II supernova SN 2020jfo in M61, implications for progenitor system, and explosion dynamics ${ }^{\star}$
}

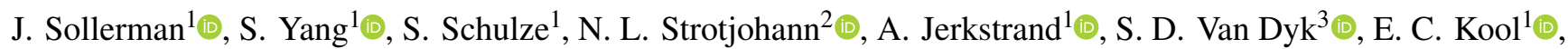

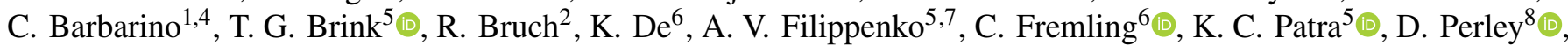

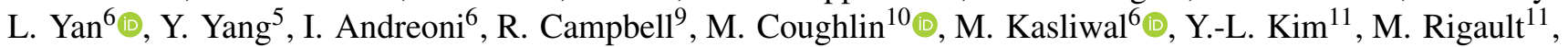

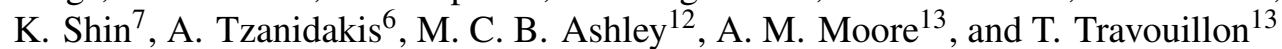

\footnotetext{
1 Department of Astronomy, The Oskar Klein Center, Stockholm University, AlbaNova 106 91, Stockholm, Sweden e-mail: jesper@astro.su.se

2 Department of Particle Physics and Astrophysics, Weizmann Institute of Science, 234 Herzl St, 76100 Rehovot, Israel

3 Caltech/IPAC, Mailcode 100-22, 1200 E. California Blvd., Pasadena, CA 91125, USA

4 The Raymond and Beverly Sackler School of Physics and Astronomy, Tel Aviv University, Tel Aviv 69978, Israel

5 Department of Astronomy, University of California, Berkeley, CA 94720-3411, USA

${ }^{6}$ Cahill Center for Astrophysics, California Institute of Technology, 1200 E. California Blvd., Pasadena, CA 91125, USA

Miller Institute for Basic Research in Science, University of California, Berkeley, CA 94720, USA

8 Astrophysics Research Institute, Liverpool John Moores University, Liverpool Science Park, 146 Brownlow Hill, Liverpool L35RF, UK

9 W. M. Keck Observatory, Kamuela, HI 96743, USA

10 School of Physics and Astronomy, University of Minnesota, Minneapolis, MN 55455, USA

11 University Claude Bernard Lyon 1, CNRS, IP2I Lyon/IN2P3, IMR 5822, 69622 Villeurbanne, France

12 School of Physics, University of New South Wales, Sydney, NSW 2052, Australia

13 Research School of Astronomy and Astrophysics, Australian National University, Canberra, ACT 2611, Australia
}

Received 24 May 2021 / Accepted 8 September 2021

\section{ABSTRACT}

\begin{abstract}
We present the discovery and extensive follow-up observations of SN 2020jfo, a Type IIP supernova (SN) in the nearby (14.5 Mpc) galaxy M61. Optical light curves (LCs) and spectra from the Zwicky Transient Facility (ZTF), complemented with data from Swift/UVOT and near-infrared photometry is presented. These were used to model the 350-day duration bolometric light curve, which exhibits a relatively short ( $\sim 65$ days) plateau. This implies a moderate ejecta mass $\left(\sim 5 M_{\odot}\right)$ at the time of explosion, whereas the deduced amount of ejected radioactive nickel is $\sim 0.025 M_{\odot}$. An extensive series of spectroscopy is presented, including spectropolarimetric observations. The nebular spectra are dominated by $\mathrm{H} \alpha$, but also reveal emission lines from oxygen and calcium. Comparisons to synthetic nebular spectra indicate an initial progenitor mass of $\sim 12 M_{\odot}$. We also note the presence of stable nickel in the nebular spectrum, and SN 2020jfo joins a small group of SNe that have inferred super-solar Ni/Fe ratios. Several years of prediscovery data were examined, but no signs of precursor activity were found. Pre-explosion Hubble Space Telescope imaging reveals a probable progenitor star, detected only in the reddest band $\left(M_{F 814 W} \approx-5.8\right)$ and it is fainter than expected for stars in the $10-15 M_{\odot}$ range. There is thus some tension between the LC analysis, the nebular spectral modeling, and the pre-explosion imaging. To compare and contrast, we present two additional core-collapse SNe monitored by the ZTF, which also have nebular $\mathrm{H} \alpha$-dominated spectra. This illustrates how the absence or presence of an interaction with circumstellar material (CSM) affect both the LCs and in particular the nebular spectra. Type II SN 2020amv has a LC powered by CSM interaction, in particular after 40 days when the LC is bumpy and slowly evolving. The late-time spectra show strong $\mathrm{H} \alpha$ emission with a structure suggesting emission from a thin, dense shell. The evolution of the complex three-horn line profile is reminiscent of that observed for SN 1998S. Finally, SN 2020jfv has a poorly constrained early-time LC, but it is of interest because of the transition from a hydrogen-poor Type IIb to a Type IIn, where the nebular spectrum after the light-curve rebrightening is dominated by $\mathrm{H} \alpha$, although with an intermediate line width.
\end{abstract}

Key words. supernovae: general

\section{Introduction}

Core-collapse (CC) supernovae ( $\mathrm{SNe}$ ) are explosions of massive stars $\left(\gtrsim 8 M_{\odot}\right)$ ending their stellar lives. The variety of CC SNe is primarily determined by the progenitor mass at the time of $\mathrm{CC}$, but also by the mass-loss history leading up to the explosion. The most common category is the hydrogen-rich class, Type II SNe.

\footnotetext{
* Full Tables 2-4 are only available at the CDS via anonymous $\mathrm{ftp}$ to cdsarc.u-strasbg. fr (130.79.128.5) or via http://cdsarc. u-strasbg.fr/viz-bin/cat/J/A+A/655/A105
}

Hydrogen-poor CC SNe also originate from massive progenitor stars, but these are stars that have lost most - or even all - of their $\mathrm{H}$ envelopes prior to explosion, including Type IIb $\mathrm{SNe}$ (some $\mathrm{H}$ left), $\mathrm{SNe} \mathrm{Ib}$ (no $\mathrm{H}$, some $\mathrm{He}$ ), and $\mathrm{SNe} \mathrm{Ic}$ (neither $\mathrm{H}$ nor $\mathrm{He}$ ); see Filippenko (1997) and Gal-Yam (2017) for reviews. There are few observational constraints on mass loss for massive stars, and the processes involved are poorly understood, but it is well established that the interaction between the ejecta and the circumstellar material (CSM) can make a significant contribution to the total SN luminosity (e.g., Chevalier \& Fransson 2017). 
In this paper, the primary aim is to present the discovery and follow-up observations of a particularly nearby supernova, the relatively normal Type II SN 2020jfo in the grand-design spiral galaxy M61, only $14.5 \mathrm{Mpc}$ away. We present the discovery by the Zwicky Transient Facility (ZTF) of this transient and the optical light curves (LCs), as well as a spectral sequence covering the first 350 days of its evolution. For comparison, we include two additional ZTF SNe that (like SN 2020jfo) are dominated by strong $\mathrm{H} \alpha$ emission in their nebular spectra. The Type II SN 2020amv has a LC dominated by CSM interaction, which also reveals itself in the complex line profiles seen in the nebular spectrum. SN 2020jfv was originally classified as a Type IIb SN (i.e., a relatively hydrogen-poor transient). However, the LC at later times starts rebrightening and the nebular spectrum is also dominated by $\mathrm{H} \alpha$, with a distinct emission-line profile. These three objects from the ZTF survey are thus used to exemplify the appearance of the nebular Balmer lines, and how these are connected to different LC shapes and powering mechanisms.

The paper is organized as follows. In Sect. 2 we present the observations, including optical photometry and spectroscopy as well as near-infrared (NIR) photometry and near-ultraviolet (UV) space-based data. Section 3 presents the similarities and differences between the objects, and Sect. 4 summarizes our conclusions and discusses our observations in context with other SNe.

\section{Observations and reductions}

\subsection{Detection and classification}

\subsubsection{SN2020jfo}

SN 2020jfo (also known as ZTF20aaynrrh) was discovered on 2020 May 6 (UT dates are used throughout this paper; first detection on JD $=2458975.70)$, with the Palomar Schmidt 48inch (P48) Samuel Oschin telescope as part of the ZTF survey (Bellm et al. 2019; Graham et al. 2019). It was reported to the Transient Name Server $\left(\mathrm{TNS}^{1}\right.$ ) on the same day (Nordin et al. 2020a), less than $2 \mathrm{~h}$ after first detection. The first detection is in the $r$ band, with a host-subtracted magnitude of $16.01 \pm 0.04$, at the J2000.0 coordinates $\alpha=12^{\mathrm{h}} 21^{\mathrm{m}} 50.48^{\mathrm{s}}, \delta=+04^{\circ} 28^{\prime} 54.1^{\prime \prime}$. The first report also mentions that the last upper limit $(g>$ 19.7 mag) from ZTF was on May 2, 4 days before discovery. Following Bruch et al. (2021), we perform a power-law fit to the early-phase $g$ and $r$ light curves, and obtain an estimated explosion date of $\mathrm{JD}_{\text {explosion }}^{\mathrm{SN200jfo}}=2458975.20$. A conservative uncertainty is ${ }_{-3.5}^{+0.5}$ days as provided by the first detection and the last nondetection. We use this as the explosion date throughout the paper, and measured the phases in rest-frame days with respect to it. This transient was subsequently also reported to the TNS by several other surveys (for example, by ATLAS and PS2 in May and by Gaia and MASTER in June), but was also bright enough to be followed by many amateur astronomers around the globe ${ }^{2}$.

SN 2020jfo is positioned in the spiral galaxy M61 (NGC 4303), which has a redshift of $z=0.00522$. It lies in the Virgo cluster and has hosted 7 known $\mathrm{SNe}$ before SN 2020jfo, the most recent being the Type Iax SN 2014dt (e.g., Kawabata et al. 2018). As is often the case, the distance of this nearby host is relatively uncertain. We follow Kawabata et al. (2018) and adopt a distance modulus of $30.81 \pm 0.20 \mathrm{mag}$, which is $14.5 \mathrm{Mpc}$. The implications for the uncertainty in the distance

\footnotetext{
1 https://wis-tns.weizmann.ac.il

2 https://www . rochesterastronomy.org/sn $202 \theta / \operatorname{sn} 2 \theta 2 \theta j$ fo. html
}

is discussed in Sect. 3.6. The position of SN 2020jfo in M61 is shown in Fig. 1.

The ZTF on-duty astronomer (J.S.) who first noticed the SN immediately triggered the robotic Palomar 60-inch telescope (P60; Cenko et al. 2006) equipped with the Spectral Energy Distribution Machine (SEDM; Blagorodnova et al. 2018). Unfortunately, the observations could not be scheduled the same night, so we instead triggered observations from La Palma. We thus classified SN 2020jfo (Perley et al. 2020a) as a Type II SN based on a spectrum obtained on 2020 May 6 with the Liverpool telescope (LT) equipped with the SPectrograph for the Rapid Acquisition of Transients (SPRAT), as well as on a spectrum from the Nordic Optical Telescope (NOT) using the Alhambra Faint Object Spectrograph (ALFOSC). These spectra were obtained 17.27 and $17.55 \mathrm{~h}$ after the first ZTF detection. The first SEDM spectrum came in a few hours thereafter, and corroborated the classification (Perley et al. 2020b).

\subsubsection{SN 2020amv}

Our first ZTF photometry of SN 2020amv (ZTF20aahbamv) was obtained on 2020 January 23 (JD = 2458871.72) with the P48. It was saved by the on-duty astronomer (J.S.) to the GROWTH Marshal (Kasliwal et al. 2019). The first detection was in the $g$ band, with a host-subtracted magnitude of $18.68 \pm 0.08$, at $\alpha=08^{\mathrm{h}} 49^{\mathrm{m}} 40.68^{\mathrm{s}}, \delta=+30^{\circ} 11^{\prime} 14.5^{\prime \prime}$ (J2000.0). The source was reported to TNS on the same day (Nordin et al. 2020b), with a note saying that the latest nondetection from ZTF was 4 days prior to discovery. This was also a ZTF discovery, but ATLAS reported the same object just a few hours later. We include the forced photometry LCs from ATLAS (Tonry et al. 2018; Smith et al. 2020) when available, for completeness. With power-law fits to the early $g$ and $r$ data, we set the explosion date as $\mathrm{JD}_{\text {explosion }}^{\mathrm{SN2020amv}}=2458871.22 \pm 0.29$.

The transient was classified as a Type II SN by ePESSTO+ (Smartt et al. 2015) using a spectrum from February 2 (Irani et al. 2020), and the SEDM spectrum from the Bright Transient Survey (Fremling et al. 2020) was also made public on TNS (Dahiwale \& Fremling 2020a). SN 2020amv was observationally well covered at early phases since it showed narrow features that could indicate CSM interaction ("flash features"; see, e.g., Gal-Yam et al. 2014; Bruch et al. 2021). The host galaxy has $z=0.0452$, and using a flat $\Lambda$ CDM cosmology with $\Omega_{\mathrm{m}}=$ 0.3 and $H_{0}=70 \mathrm{~km} \mathrm{~s}^{-1} \mathrm{Mpc}^{-1}$ this corresponds to $200 \mathrm{Mpc}$ (distance modulus $36.5 \mathrm{mag}$ ).

\subsubsection{SN 2020jfv}

SN 2020jfv (ZTF20abgbuly) was first reported to TNS by ATLAS (Tonry et al. 2020), with a detection on 2020 May 5. Gaia actually detected the transient earlier (on April 30), and claimed a nondetection the previous night. With ZTF the first observations were obtained later (June 18), when the object was already clearly declining. For SN 2020jfv we adopt 2020 April $30\left(\mathrm{JD}_{\text {explosion }}^{\mathrm{SN2020j \textrm {fv }}}=2458969.51\right)$ as both the discovery date and the explosion date, but we note that there is quite some uncertainty in the actual date of explosion. The object is positioned at $\alpha=23^{\mathrm{h}} 06^{\mathrm{m}} 35.75^{\mathrm{s}}, \delta=+00^{\circ} 36^{\prime} 43.7^{\prime \prime}$ (J2000.0), not far from the center of the face-on spiral galaxy WISEA J230635.97+003641.9. This galaxy had no previously reported redshift, and our estimate of $z=0.017$ comes from the measured host-galaxy narrow emission lines in our latetime nebular spectra. The distance is thus estimated to be $73.8 \mathrm{Mpc}$ adopting the same cosmology as provided above. 

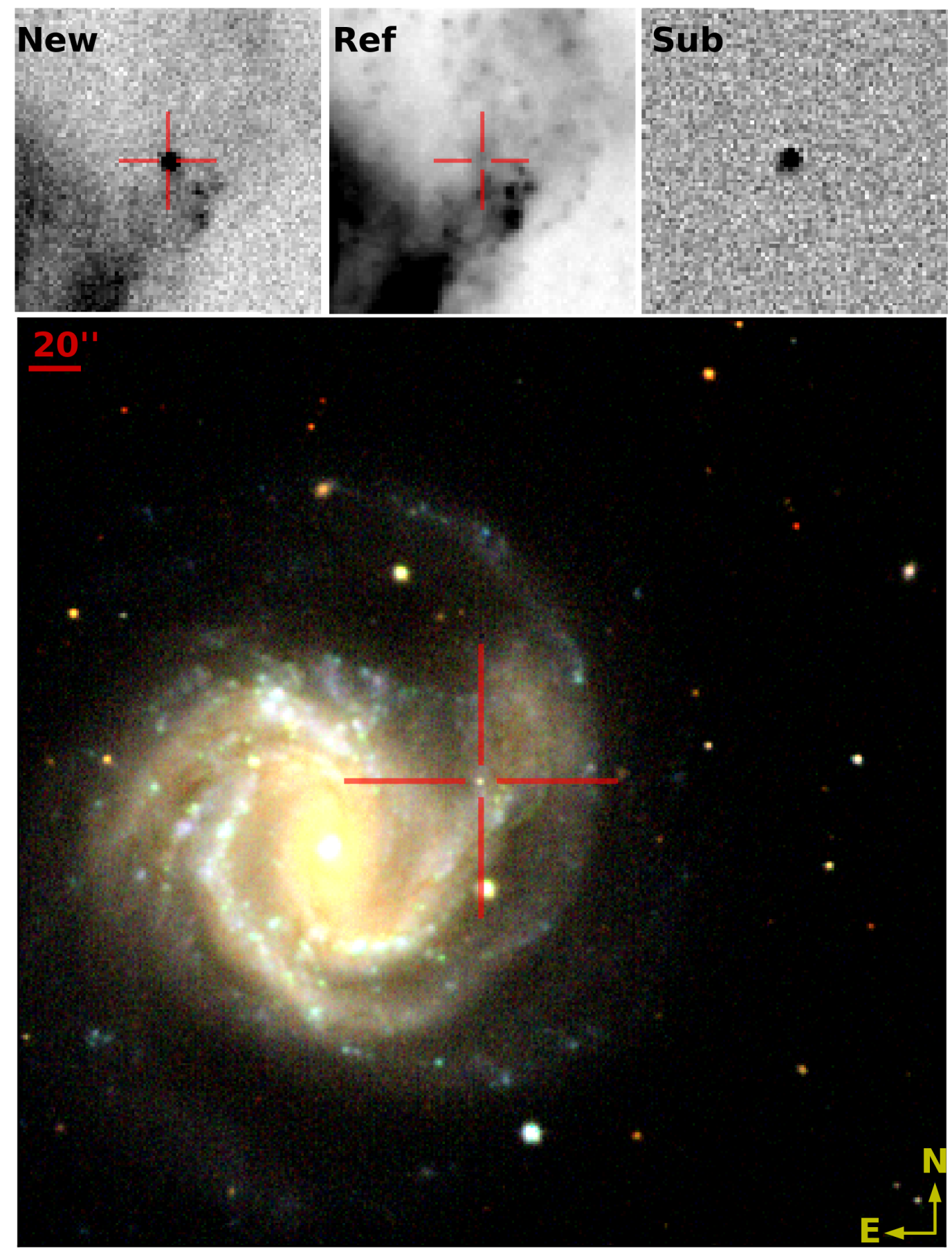

Fig. 1. SN2020jfo in M 61. The $r$-band image subtraction is shown in the top panels, with the SN image observed on 2020 May 6 on the left; this is the (ZTF) discovery frame. The SN position is marked. Middle top panel: reference image (the template), and right panel: SN standing out in the image subtraction. Bottom panel: gri-color composite image of the host galaxy and its environment. It was composed of ZTF $g$, $r$, and $i$ images of the field observed on 2020 December 21, 7 months after the first ZTF detection. The SN is still visible and marked by the red cross.

The SN was classified as Type IIb based on an SEDM spectrum obtained on June 20 (Dahiwale \& Fremling 2020b). It continued to fade in a linear fashion for the next 100 days, but it thereafter began rebrightening, especially in the $r$ band. Late-time spectra demonstrate that this is due to CSM interaction driving a conspicuous nebular $\mathrm{H} \alpha$ line. The transformation from a stripped-envelope SN to a CSM interacting Type IIn is reminiscent of the evolution discussed by for example Sollerman et al. (2020), Milisavljevic et al. (2015),
Mauerhan et al. (2018), Prentice et al. (2020), Chandra et al. (2020), and Tartaglia et al. (2021). Some of the data for these three $\mathrm{SNe}$ and their host galaxies is summarized in Table 1.

\subsection{Optical photometry}

Following the discoveries as outlined above, ZTF obtained regular follow-up photometry in the $g, r$, and sometimes $i$ bands with the ZTF camera (Dekany et al. 2020) on the P48. Additional 
Table 1. Supernova and host-galaxy properties.

\begin{tabular}{lccccccc}
\hline \hline IAU name & ZTF name & $\begin{array}{c}\text { Explosion date } \\
(\mathrm{JD})\end{array}$ & SN type & Redshift & Host & $\begin{array}{c}\text { Distance } \\
(\mathrm{Mpc})\end{array}$ & $\begin{array}{c}E(B-V)_{\mathrm{MW}} \\
(\mathrm{mag})\end{array}$ \\
\hline SN 2020amv & ZTF20aahbamv & 2458871.22 & IIn & 0.0452 & WISEA J084940.38+301115.5 & 200.3 & 0.032 \\
SN 2020jfo & ZTF20aaynrrh & 2458975.20 & II & 0.00522 & M 61 & 14.5 & 0.020 \\
SN 2020jfv & ZTF20abgbuly & 2458969.51 & IIb $\rightarrow$ IIn & 0.017 & WISEA J230635.97+003641.9 & 73.8 & 0.045 \\
\hline
\end{tabular}

Table 2. Summary of photometry for SN 2020jfo.

\begin{tabular}{|c|c|c|c|c|c|c|c|}
\hline $\begin{array}{l}\text { Observation } \\
\text { date (JD) }\end{array}$ & $\begin{array}{c}\text { Rest frame } \\
\text { phase (days) }\end{array}$ & Filter & $\begin{array}{c}\text { Absolute mag } \\
\text { (mag) }\end{array}$ & $\begin{array}{c}\text { Apparent mag } \\
\text { (mag) }\end{array}$ & $\begin{array}{c}\text { Limiting mag } \\
\text { (mag) }\end{array}$ & $\begin{array}{c}\text { Mag error } \\
(\mathrm{mag})\end{array}$ & Instrument \\
\hline 2458964.87 & -10.3 & $o$ & 99.00 & 99.00 & 20.00 & 99.00 & ATLAS \\
\hline 2458965.73 & -9.4 & $g$ & 99.00 & 99.00 & 21.59 & 99.00 & P48 \\
\hline 2458965.79 & -9.4 & $r$ & 99.00 & 99.00 & 21.56 & 99.00 & P48 \\
\hline 2458966.89 & -8.3 & $c$ & 99.00 & 99.00 & 20.86 & 99.00 & ATLAS \\
\hline 2458967.75 & -7.4 & $g$ & 99.00 & 99.00 & 21.32 & 99.00 & P48 \\
\hline 2458968.70 & -6.5 & $r$ & 99.00 & 99.00 & 21.38 & 99.00 & P48 \\
\hline 2458968.77 & -6.4 & $g$ & 99.00 & 99.00 & 21.37 & 99.00 & P48 \\
\hline 2458969.89 & -5.3 & $o$ & 99.00 & 99.00 & 20.97 & 99.00 & ATLAS \\
\hline 2458971.71 & -3.5 & $r$ & 99.00 & 99.00 & 20.83 & 99.00 & P48 \\
\hline 2458971.78 & -3.4 & $g$ & 99.00 & 99.00 & 20.76 & 99.00 & P48 \\
\hline 2458975.70 & 0.5 & $r$ & -14.88 & 15.99 & 20.03 & 0.01 & P48 \\
\hline 2458975.75 & 0.5 & $g$ & -15.47 & 15.40 & 19.84 & 0.01 & P48 \\
\hline 2458976.37 & 1.2 & $z$ & -15.17 & 15.70 & 20.54 & 0.01 & $\mathrm{LT}$ \\
\hline 2458976.37 & 1.2 & $\ddot{i}$ & -15.38 & 15.49 & 20.82 & 0.03 & LT \\
\hline 2458976.37 & 1.2 & $r$ & -15.62 & 15.25 & 20.40 & 0.02 & LT \\
\hline 2458976.37 & 1.2 & $g$ & -15.87 & 15.00 & 20.66 & 0.05 & $\mathrm{LT}$ \\
\hline 2458976.37 & 1.2 & $u$ & -16.22 & 14.65 & 18.00 & 0.07 & $\mathrm{LT}$ \\
\hline 2458976.56 & 1.4 & $z$ & -15.25 & 15.62 & 20.20 & 0.01 & $\mathrm{LT}$ \\
\hline 2458976.56 & 1.4 & $i$ & -15.48 & 15.39 & 20.31 & 0.01 & $\mathrm{LT}$ \\
\hline 2458976.56 & 1.4 & $r$ & -15.68 & 15.19 & 20.39 & 0.01 & $\mathrm{LT}$ \\
\hline 2458976.56 & 1.4 & $g$ & -16.10 & 14.77 & 20.25 & 0.01 & $\mathrm{LT}$ \\
\hline 2458976.56 & 1.4 & $u$ & -16.30 & 14.57 & 18.00 & 0.07 & $\mathrm{LT}$ \\
\hline 2458976.70 & 1.5 & $r$ & -15.87 & 15.00 & 99.00 & 0.02 & P60 \\
\hline 2458976.72 & 1.5 & $r$ & -15.89 & 14.98 & 99.00 & 0.10 & P60 \\
\hline 2458976.73 & 1.5 & $g$ & -16.04 & 14.83 & 99.00 & 0.03 & P60 \\
\hline 2458976.73 & 1.5 & $i$ & -15.69 & 15.18 & 99.00 & 0.04 & P60 \\
\hline
\end{tabular}

Notes. The full table is available at the CDS.

images were obtained with the LT and with the P60. Some late-time photometry was also obtained with ALFOSC on the NOT. P48 LCs come from the ZTF pipeline (Masci et al. 2019), where we have applied forced photometry (see, e.g., Yao et al. 2019). Photometry from the P60 and LT was produced with the image-subtraction pipeline described by Fremling et al. (2016), with template images from the Sloan Digital Sky Survey (SDSS; Ahn et al. 2014). This pipeline produces point-spread-function (PSF) magnitudes, calibrated against SDSS stars in the field. The NOT photometry was done using template subtractions performed with hotpants ${ }^{3}$, using archival SDSS images as templates. The magnitudes of the transient were measured using $\mathrm{SNOoPY}^{4}$ and calibrated against SDSS stars in the field. All magnitudes are reported in the AB system (Oke \& Gunn 1983).

\footnotetext{
3 http://www.astro.washington.edu/users/becker/v2.0/ hotpants.html

4 SNOoPy is a package for SN photometry using PSF fitting and template subtraction developed by E. Cappellaro. A package description can be found at http://sngroup.oapd.inaf.it/snoopy.html
}

For completeness and comparison, we have also included data from the forced photometry service from ATLAS. Those data points are included in the figures, but are generally not part of the analysis and measurements.

In our analysis we have corrected all photometry for Galactic extinction, using the Milky Way (MW) color excess $E(B-V)_{\mathrm{MW}}$ toward the position of the $\mathrm{SNe}$, as provided in Table 1 . All reddening corrections are applied using the Cardelli et al. (1989) extinction law with $R_{V}=3.1$. No further host-galaxy extinction has been applied. We discuss the effects of this assumption in Sect. 3.6. All the photometry is given in Tables 2-4. The three light curves are shown in Fig. 2.

\subsection{Near-infrared photometry}

SN 2020jfo was observed in the NIR $J$ band as a part of regular survey operations of the Palomar Gattini-IR (PGIR) survey. PGIR is a wide-field NIR survey at Palomar Observatory, observing the entire visible sky at a median cadence of $\sim 2$ days, and to a median $5 \sigma$ depth of $J \approx 15.7 \mathrm{AB}$ mag 
Table 3. Summary of photometry for SN 2020amv.

\begin{tabular}{lccccccc}
\hline \hline $\begin{array}{l}\text { Observation } \\
\text { date (JD) }\end{array}$ & $\begin{array}{c}\text { Rest frame } \\
\text { phase (days) }\end{array}$ & Filter & $\begin{array}{c}\text { Absolute mag } \\
(\mathrm{mag})\end{array}$ & $\begin{array}{c}\text { Apparent mag } \\
(\mathrm{mag})\end{array}$ & $\begin{array}{c}\text { Limiting mag } \\
(\mathrm{mag})\end{array}$ & $\begin{array}{c}\text { Mag error } \\
(\mathrm{mag})\end{array}$ & Instrument \\
\hline 2458861.80 & -9.0 & $r$ & 99.00 & 99.00 & 20.22 & 99.00 & P48 \\
2458861.85 & -9.0 & $g$ & 99.00 & 99.00 & 20.21 & 99.00 & P48 \\
2458863.85 & -7.0 & $r$ & 99.00 & 99.00 & 21.07 & 99.00 & P48 \\
2458864.70 & -6.2 & $g$ & 99.00 & 99.00 & 21.60 & 99.00 & P48 \\
2458864.74 & -6.2 & $r$ & 99.00 & 99.00 & 21.65 & 99.00 & P48 \\
2458867.90 & -3.2 & $g$ & 99.00 & 99.00 & 21.72 & 99.00 & P48 \\
2458869.94 & -1.2 & $o$ & 99.00 & 99.00 & 20.84 & 99.00 & ATLAS \\
2458871.72 & 0.5 & $g$ & -17.90 & 18.71 & 21.17 & 0.04 & P48 \\
2458871.73 & 0.5 & $g$ & -17.89 & 18.72 & 21.22 & 0.04 & P48 \\
2458871.77 & 0.5 & $r$ & -17.59 & 19.02 & 21.37 & 0.04 & P48 \\
2458871.77 & 0.5 & $r$ & -17.57 & 19.04 & 21.35 & 0.04 & P48 \\
2458871.78 & 0.5 & $r$ & -17.61 & 19.00 & 21.30 & 0.04 & P48 \\
2458871.78 & 0.5 & $r$ & -17.54 & 19.07 & 21.32 & 0.05 & P48 \\
2458871.89 & 0.6 & $c$ & -17.85 & 18.76 & 20.96 & 0.18 & ATLAS \\
2458872.74 & 1.4 & $g$ & -18.50 & 18.11 & 21.19 & 0.02 & P48 \\
2458872.78 & 1.5 & $r$ & -18.18 & 18.43 & 21.25 & 0.03 & P48 \\
\hline
\end{tabular}

Notes. The full table is available at the CDS.

Table 4. Summary of photometry for SN 2020jfv.

\begin{tabular}{|c|c|c|c|c|c|c|c|}
\hline $\begin{array}{l}\text { Observation } \\
\text { date (JD) }\end{array}$ & $\begin{array}{c}\text { Rest frame } \\
\text { phase (days) }\end{array}$ & Filter & $\begin{array}{c}\text { Absolute mag } \\
\text { (mag) }\end{array}$ & $\begin{array}{c}\text { Apparent mag } \\
(\mathrm{mag})\end{array}$ & $\begin{array}{c}\text { Limiting mag } \\
\text { (mag) }\end{array}$ & $\begin{array}{c}\text { Mag error } \\
(\mathrm{mag})\end{array}$ & Instrument \\
\hline 2458860.63 & -107.1 & $r$ & 99.00 & 99.00 & 20.10 & 99.00 & P48 \\
\hline 2458863.59 & -104.1 & $r$ & 99.00 & 99.00 & 20.63 & 99.00 & P48 \\
\hline 2458863.61 & -104.1 & $g$ & 99.00 & 99.00 & 20.74 & 99.00 & P48 \\
\hline 2458872.59 & -95.3 & $r$ & 99.00 & 99.00 & 20.13 & 99.00 & P48 \\
\hline 2458872.60 & -95.3 & $r$ & 99.00 & 99.00 & 20.33 & 99.00 & P48 \\
\hline 2458872.60 & -95.3 & $r$ & 99.00 & 99.00 & 20.27 & 99.00 & P48 \\
\hline 2458872.61 & -95.3 & $r$ & 99.00 & 99.00 & 20.05 & 99.00 & P48 \\
\hline 2458872.72 & -95.2 & $o$ & 99.00 & 99.00 & 20.11 & 99.00 & ATLAS \\
\hline 2458874.60 & -93.3 & $r$ & 99.00 & 99.00 & 19.31 & 99.00 & $\mathrm{P} 48$ \\
\hline 2458874.60 & -93.3 & $r$ & 99.00 & 99.00 & 19.40 & 99.00 & $\mathrm{P} 48$ \\
\hline 2458874.61 & -93.3 & $r$ & 99.00 & 99.00 & 19.87 & 99.00 & P48 \\
\hline 2458874.61 & -93.3 & $r$ & 99.00 & 99.00 & 19.15 & 99.00 & P48 \\
\hline 2458874.72 & -93.2 & $c$ & 99.00 & 99.00 & 19.30 & 99.00 & ATLAS \\
\hline 2458876.60 & -91.4 & $r$ & 99.00 & 99.00 & 18.24 & 99.00 & P48 \\
\hline 2458876.60 & -91.4 & $r$ & 99.00 & 99.00 & 18.74 & 99.00 & P48 \\
\hline 2458876.61 & -91.4 & $r$ & 99.00 & 99.00 & 17.64 & 99.00 & P48 \\
\hline 2458975.11 & 5.5 & $o$ & -16.89 & 17.59 & 19.71 & 0.06 & ATLAS \\
\hline 2458999.09 & 29.1 & $c$ & -15.95 & 18.53 & 20.83 & 0.14 & ATLAS \\
\hline 2459003.10 & 33.0 & $c$ & -15.77 & 18.71 & 20.93 & 0.17 & ATLAS \\
\hline 2459005.06 & 35.0 & $o$ & -16.42 & 18.06 & 20.01 & 0.09 & ATLAS \\
\hline 2459009.09 & 38.9 & $o$ & 99.00 & 99.00 & 17.16 & 99.00 & ATLAS \\
\hline 2459018.89 & 48.6 & $g$ & -15.02 & 19.45 & 21.24 & 0.07 & $\mathrm{P} 48$ \\
\hline 2459018.96 & 48.6 & $r$ & -16.15 & 18.33 & 21.25 & 0.02 & P48 \\
\hline
\end{tabular}

Notes. The full table is available at the CDS.

(Moore \& Kasliwal 2019; De et al. 2020a). The transient was clearly detected in the PGIR data for $\sim 30$ days after discovery. We derived $J$-band photometry (calibrated to the 2MASS catalogue in the Vega system) of SN 2020jfo by performing forced PSF photometry at the location of the transient on the PGIR difference images, using the method described by De et al. (2020b). The photometry is given in Table 5 and included in Fig. 2.

\subsection{Swift observations}

\subsubsection{UVOT photometry}

For SN 2020jfo, we also have observations in the ultraviolet (UV) and optical from the UV Optical Telescope onboard the Neil Gehrels Swift observatory (UVOT; Gehrels et al. 2004; Roming et al. 2005). As shown in Table 6, 25 epochs were 


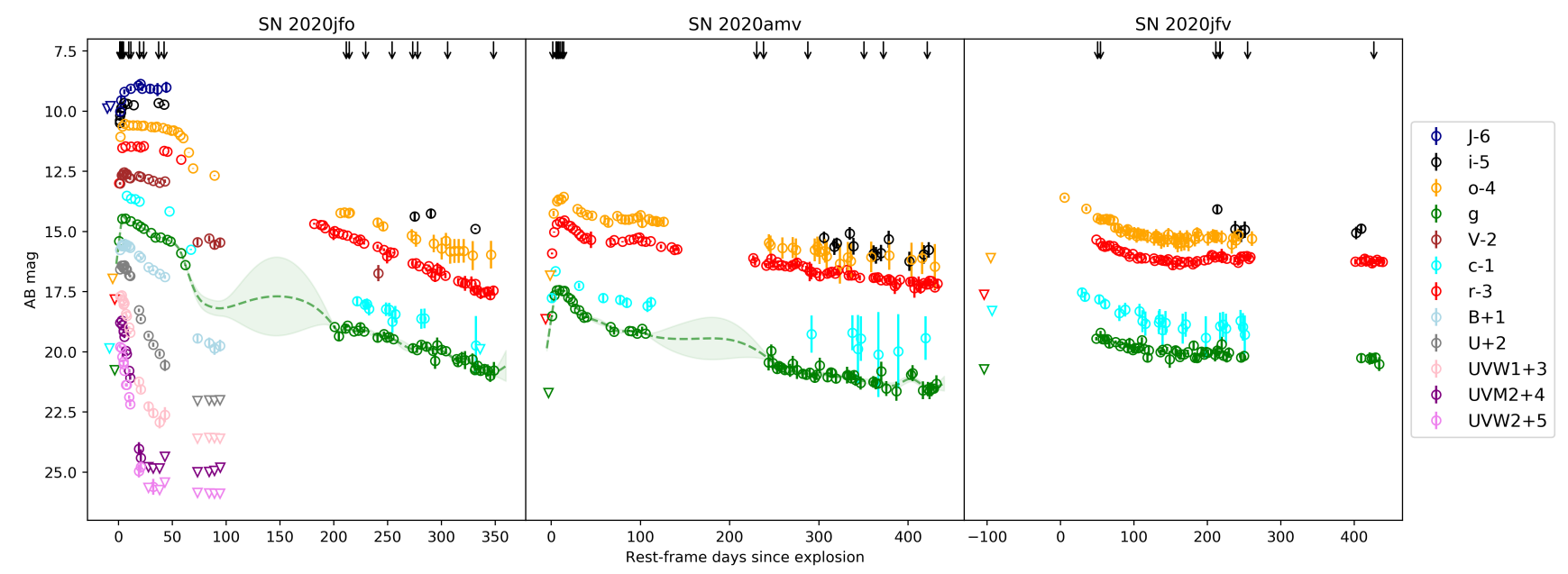

Fig. 2. Light curves of the three supernovae. Left: SN 2020jfo in multiple bands with symbols and offsets as provided in the legend to the very right. In this case it includes six passbands from Swift and three from ZTF and LT, as well as near-infrared $J$-band data. These are observed (AB) magnitudes plotted versus rest-frame time in days since explosion. For the $J$-band data, the Vega/AB magnitude conversion follows Blanton \& Roweis (2007). ATLAS forced photometry in the $c$ and $o$ bands is also included, and the ZTF and ATLAS data are in 3 day bins. The arrows on top indicate the epochs of spectroscopy, and the lines with error regions are Gaussian Process estimates of the interpolated LCs (for $g$ band). Middle and right panels: LCs for SN 2020amv and SN 2020jfv, respectively.

Table 5. Summary of PGIR $J$-band observations of SN 2020jfo.

\begin{tabular}{lcc}
\hline $\begin{array}{l}\text { Observation date } \\
\text { (JD) }\end{array}$ & $\begin{array}{c}\text { Rest-frame phase } \\
\text { (days) }\end{array}$ & $\begin{array}{c}J \\
(\mathrm{mag})\end{array}$ \\
\hline 2458635.71 & -328.22 & $>14.87$ \\
2458640.73 & -323.28 & $>15.17$ \\
2458648.71 & -315.44 & $>15.12$ \\
2458669.68 & -294.82 & $>14.84$ \\
2458676.68 & -287.94 & $>14.87$ \\
2458682.67 & -282.05 & $>14.84$ \\
2458866.99 & -100.81 & $>14.82$ \\
2458896.06 & -72.22 & $>14.98$ \\
2458913.02 & -55.55 & $>14.82$ \\
2458964.87 & -4.56 & $>14.98$ \\
2458967.87 & -1.61 & $>14.88$ \\
2458976.81 & 7.18 & $15.10 \pm 0.23$ \\
2458977.83 & 8.18 & $14.64 \pm 0.18$ \\
2458980.74 & 11.04 & $14.29 \pm 0.10$ \\
2458986.74 & 16.94 & $14.15 \pm 0.10$ \\
2458993.75 & 23.83 & $14.02 \pm 0.10$ \\
2458995.68 & 25.73 & $13.96 \pm 0.08$ \\
2458997.23 & 27.26 & $14.16 \pm 0.09$ \\
2459004.77 & 34.67 & $14.15 \pm 0.19$ \\
2459011.75 & 41.53 & $14.19 \pm 0.27$ \\
2459019.72 & 49.37 & $14.10 \pm 0.23$ \\
2459177.06 & 204.08 & $>14.79$ \\
2459223.00 & 249.25 & $>14.18$ \\
2459249.93 & 275.73 & $>14.49$ \\
2459269.87 & 295.34 & $>14.86$ \\
2459280.84 & 306.13 & $>15.17$ \\
\hline
\end{tabular}

Notes. Fluxes with $S / N<3 \sigma$ are shown as upper limits. These magnitudes are Vega tied to 2MASS, and $m(\mathrm{AB})-m($ Vega $)=0.91$.

obtained over the first 300 days. Our first Swift/UVOT observation was performed on 2020 May 7 (just 1.4 days after estimated explosion), and provided detections in all bands.
The brightness in the UVOT filters was measured with UVOT-specific tools in HEAsoft ${ }^{5}$. Source counts were extracted from the images using a circular aperture with a radius of $3^{\prime \prime}$. The background was estimated using a significantly larger circular region. The count rates were measured from the images using the Swift tool uvotsource. They were converted to magnitudes using the UVOT photometric zero points (Breeveld et al. 2011) and the latest calibration files from 2020 September. To remove the host-galaxy contribution, we used observations from before the SN explosion. We measured the flux at the SN site using the same source and background apertures and arithmetically subtracted the host contribution from the SN photometry. All magnitudes were transformed into the $\mathrm{AB}$ system using Breeveld et al. (2011). These measurements are included in Fig. 2.

\subsubsection{X-rays}

The field of SN2020jfo was observed with Swift's onboard X-ray telescope (XRT; Burrows et al. 2005) in photon-counting mode several times between 2020 May 7 and 2021 January 30. Swift also observed this field between 2008 and 2016, long before the SN explosion. We analyzed all data with the online tools of the UK Swift team ${ }^{6}$ that use the methods described by Evans et al. (2007, 2009) and the software package HEAsoft.

We detected emission at the SN position in the 2008-2016 datasets. To recover any SN flux in the later data, we numerically subtracted that baseline flux, but this did not result in any significant detections $(<1.4 \sigma)$. To convert count-rate limits to flux, we use WebPIMMS ${ }^{7}$, assume a power law with a photon index of 2 and a Galactic equivalent neutral-hydrogen column density of $1.58 \times 10^{20} \mathrm{~cm}^{-2}$. We conclude that any X-ray emission from SN 2020jfo must be fainter than a few $10^{39} \mathrm{erg} \mathrm{s}^{-1}$.

\footnotetext{
5 https://heasarc.gsfc.nasa.gov/docs/software/heasoft/ version 6.26.1.

6 https://www.swift.ac.uk/user_objects/

7 https://heasarc.gsfc.nasa.gov/cgi-bin/Tools/w3pimms/ w3pimms.pl
} 
Table 6. Summary of Swift/UVOT observations of SN 2020jfo.

\begin{tabular}{|c|c|c|c|c|c|c|c|}
\hline $\begin{array}{l}\text { Observatin date } \\
\text { (MJD) }\end{array}$ & $\begin{array}{c}\text { Rest-frame phase } \\
\text { (days) }\end{array}$ & $\begin{array}{c}V \\
(\mathrm{mag})\end{array}$ & $\begin{array}{c}B \\
(\mathrm{mag}) \\
\end{array}$ & $\begin{array}{c}U \\
(\mathrm{mag})\end{array}$ & $\begin{array}{c}U V W 1 \\
(\mathrm{mag})\end{array}$ & $\begin{array}{c}U V W 2 \\
(\mathrm{mag})\end{array}$ & $\begin{array}{c}U V M 2 \\
\text { (mag) }\end{array}$ \\
\hline 2458976.62 & 1.41 & $15.00(0.06)$ & $14.77(0.04)$ & $14.57(0.05)$ & $14.69(0.05)$ & $14.79(0.06)$ & $14.78(0.05)$ \\
\hline 2458978.23 & 3.02 & $14.66(0.06)$ & $14.57(0.05)$ & $14.41(0.05)$ & $14.65(0.05)$ & $14.85(0.06)$ & $14.71(0.05)$ \\
\hline 2458978.73 & 3.51 & $14.66(0.06)$ & $14.61(0.05)$ & $14.43(0.05)$ & $14.83(0.05)$ & $15.21(0.07)$ & $14.89(0.05)$ \\
\hline 2458979.85 & 4.63 & $14.55(0.06)$ & $14.51(0.05)$ & $14.41(0.05)$ & $14.90(0.05)$ & $15.41(0.07)$ & $15.12(0.05)$ \\
\hline 2458979.92 & 4.70 & $14.63(0.07)$ & $14.59(0.05)$ & $14.51(0.05)$ & $15.03(0.05)$ & $15.51(0.07)$ & $15.17(0.05)$ \\
\hline 2458980.72 & 5.49 & $14.54(0.05)$ & $14.51(0.05)$ & $14.41(0.05)$ & $14.98(0.05)$ & $15.79(0.07)$ & $15.38(0.05)$ \\
\hline 2458982.26 & 7.02 & $14.62(0.06)$ & $14.63(0.05)$ & $14.57(0.05)$ & $15.42(0.05)$ & $16.37(0.07)$ & $15.96(0.05)$ \\
\hline 2458982.92 & 7.68 & $14.61(0.06)$ & $14.55(0.05)$ & $14.58(0.05)$ & $15.51(0.05)$ & $16.38(0.07)$ & $16.09(0.06)$ \\
\hline 2458985.17 & 9.92 & $14.77(0.06)$ & $14.61(0.05)$ & $14.81(0.05)$ & $15.99(0.06)$ & $16.88(0.08)$ & $16.78(0.06)$ \\
\hline 2458986.16 & 10.91 & $14.78(0.08)$ & $14.68(0.06)$ & $14.85(0.06)$ & $16.21(0.07)$ & $17.18(0.09)$ & $17.08(0.08)$ \\
\hline 2458994.41 & 19.11 & $14.69(0.06)$ & $15.00(0.05)$ & $16.27(0.07)$ & $18.24(0.12)$ & $19.96(0.27)$ & $20.03(0.27)$ \\
\hline 2458996.06 & 20.75 & $14.72(0.06)$ & $15.09(0.06)$ & $16.63(0.08)$ & $18.56(0.14)$ & $19.79(0.24)$ & $20.41(0.36)$ \\
\hline 2459003.26 & 27.91 & $14.82(0.05)$ & $15.49(0.05)$ & $17.33(0.08)$ & $19.27(0.17)$ & $>20.66$ & $>20.80$ \\
\hline 2459007.61 & 32.24 & $14.89(0.05)$ & $15.61(0.05)$ & $17.70(0.09)$ & $19.54(0.19)$ & $20.61(0.33)$ & $>20.85$ \\
\hline 2459013.59 & 38.19 & $14.97(0.05)$ & $15.76(0.05)$ & $18.08(0.11)$ & $19.92(0.26)$ & $>20.74$ & $>20.84$ \\
\hline 2459018.49 & 43.07 & $14.91(0.07)$ & $15.89(0.08)$ & $18.55(0.24)$ & $19.61(0.32)$ & $>20.43$ & $>20.36$ \\
\hline 2459048.91 & 73.33 & $17.45(0.14)$ & $18.44(0.19)$ & $>20.05$ & $>20.62$ & $>20.87$ & $>21.00$ \\
\hline 2459059.83 & 84.19 & $17.29(0.13)$ & $18.64(0.23)$ & $>20.02$ & $>20.58$ & $>20.88$ & $>20.99$ \\
\hline 2459064.91 & 89.24 & $17.55(0.16)$ & $18.86(0.27)$ & $>20.03$ & $>20.59$ & $>20.90$ & $>20.95$ \\
\hline 2459070.00 & 94.31 & $17.46(0.20)$ & $18.75(0.25)$ & $>20.01$ & $>20.61$ & $>20.89$ & $>20.81$ \\
\hline 2459168.08 & 191.88 & $>18.06$ & $>19.08$ & $>19.85$ & $>20.49$ & $>20.72$ & $>20.77$ \\
\hline 2459195.53 & 219.19 & $>18.52$ & $>19.19$ & $>19.91$ & $>20.54$ & $>20.82$ & $>20.91$ \\
\hline 2459207.91 & 231.50 & $>18.81$ & $>19.43$ & $>20.15$ & $>20.67$ & $>20.95$ & $>21.02$ \\
\hline 2459217.75 & 241.29 & $18.73(0.34)$ & $>19.46$ & $>20.18$ & $>20.64$ & $>20.99$ & $>21.04$ \\
\hline 2459228.02 & 251.51 & $>18.84$ & $>19.45$ & $>20.21$ & $>20.66$ & $>20.99$ & $>21.07$ \\
\hline
\end{tabular}

Notes. Fluxes with $S / N<3 \sigma$ are shown as upper limits.

\subsection{Pre-explosion imaging}

\subsubsection{Progenitor}

An explosion in a nearby Messier galaxy could allow for an investigation of the site of the progenitor star. Fortunately, the site of SN 2020jfo was serendipitously imaged prior to explosion with the Hubble Space Telescope (HST) Advanced Camera for Surveys (ACS)/Wide Field Channel (WFC) in bands $F 435 W$ and $F 814 W$ (1090s total exposure time in each band) on 2012 May $24^{8}$ and in $F 814 W\left(2112\right.$ s) on 2019 April $7^{9}$, as well as with the Wide Field Camera 3 (WFC3)/UVIS channel on 2020 March $29^{10}$ in $F 275 W(2190$ s), F336W (1110 s), $F 438 W(1050 \mathrm{~s}), F 555 W(670 \mathrm{~s})$, and $F 814 W(803 \mathrm{~s})$. The SN site is also located in archival HST Wide-Field Planetary Camera 2 (WFPC2) data, but owing to the comparatively inferior spatial resolution and sensitivity of the WFPC2 data, we do not consider them further.

To locate the precise position of the SN in the archival HST datasets, we obtained high-spatial-resolution images in the $K^{\prime}$ band with the adaptive optics (AO)-assisted OSIRIS Imager (Larkin et al. 2006) on the Keck-I 10 m telescope on 2020 July 7. The SN field required laser-guide-star AO and short-turnaround clearance for satellite avoidance. There were 10 frames added together, with each coadd consisting of 8 dithered images of duration $14.75 \mathrm{~s}$, so the total integration time was $1180 \mathrm{~s}$.

\footnotetext{
8 GO-12574; PI D. Leonard.

9 GO-15645; PI D. Sand.

${ }^{10}$ GO-15654; PI J. Lee.
}

We astrometrically registered the Keck AO image to the 2012 ACS/WFC F $814 W$ image using 32 stars in common between the two image datasets, employing the task geomap in PyRAF with parameter "calctype" set to "double". The geometric distortion for the OSIRIS AO imager is quite small ( $<5$ milliarcsec), so a distortion correction is not strictly required ${ }^{11}$. We were able to achieve a satisfactory solution to a precision of 0.15 WFC pixel (7.5 milliarcsec). Figure 3 shows the Keck and ACS images in a broader view, as well as a zoom-in on the SN site. With regards to a version of the $2012 \mathrm{~F} 814 \mathrm{~W}$ image mosaic available from the Hubble Legacy Archive ${ }^{12}$, from the position of the SN in the OSIRIS image, and referencing our astrometric solution, we expect the precise SN location at pixel $(2110.05,3062.02)$. In the image mosaic a faint object can be seen with a centroid position of $(2110.06,3061.93)$. The difference between these two pixel values is within the astrometric uncertainty, and we therefore consider the object to very likely be the candidate for the $\mathrm{SN}$ progenitor.

In order to determine the brightness of this object from the archival HST data, we extracted photometry from the individual frames for both the ACS and WFC3 observations with Dolphot (Dolphin 2016). We used recommended parameters for Dolphot appropriate for a crowded extragalactic environment $^{13}$. The object is only detected in $F 814 W$. We measured $F 814 W=25.02 \pm 0.07 \mathrm{mag}$ in 2021 , and $25.56 \pm 0.09$ and $25.08 \pm 0.13 \mathrm{mag}$ in 2019 and 2020, respectively. Upper limits

\footnotetext{
11 J. Lu, priv. comm.

12 http://hla.stsci.edu/

${ }^{13}$ FitSky $=3$ and RAper $=8$.
} 

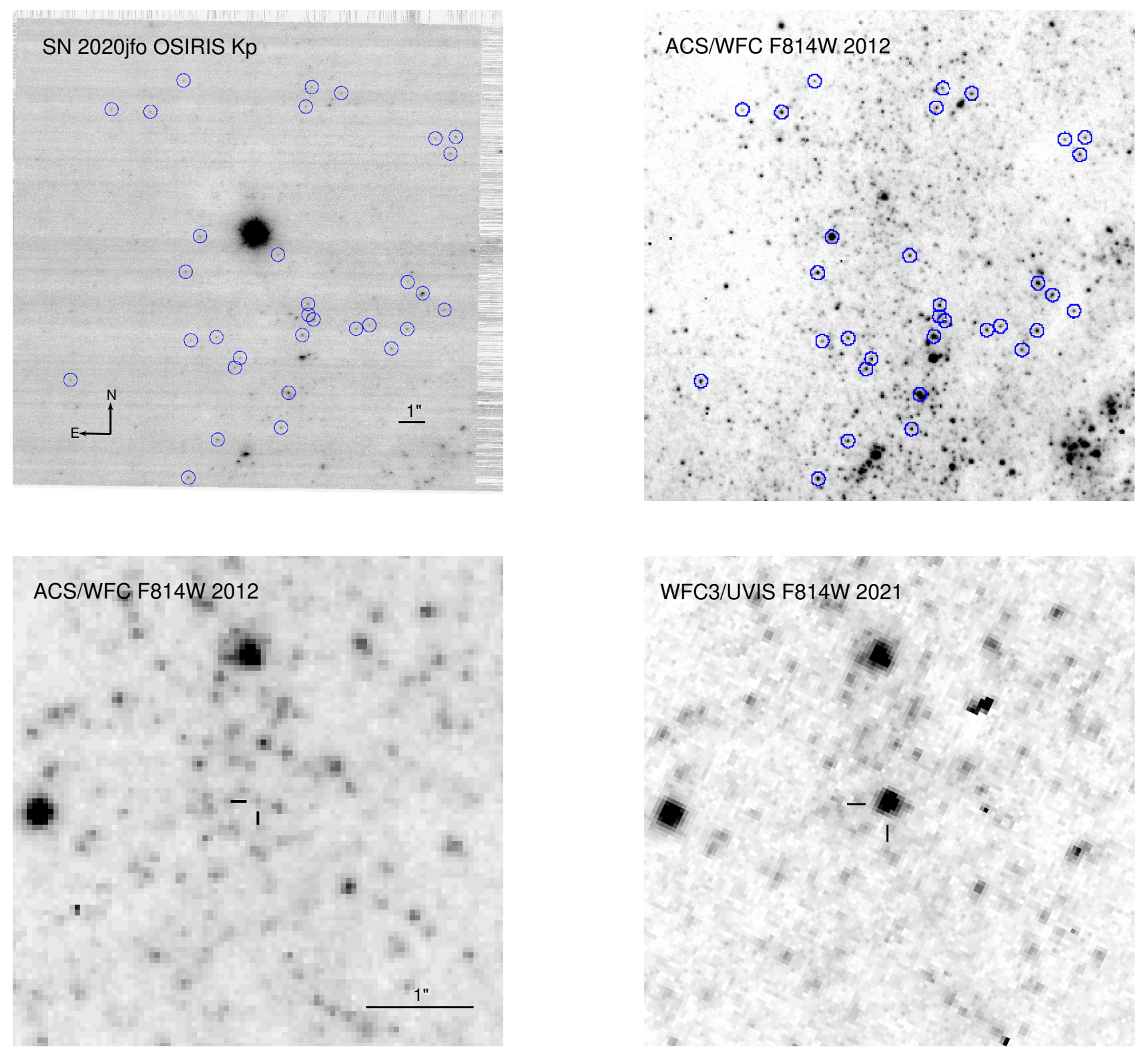

Fig. 3. Top left: adaptive optics (AO) image of SN 2020jfo obtained with the OSIRIS imager on the Keck-I $10 \mathrm{~m}$ telescope in the $K^{\prime}$ band on 2020 July 7. Top right: portion of an archival HST ACS/WFC image mosaic obtained in F814W on 2012 May 24, which contains the SN site, shown at the same scale and orientation as the top-left panel. In both top panels, the 32 stars in common between the two images and used in the astrometric registration are circled. Bottom left: zoom-in on the SN site in the HST image, with the SN progenitor candidate indicated by tick marks. Bottom right: zoom-in on the SN site after the explosion, in a HST WFC3/UVIS F814W image from 2021 July 28 . The SN is clearly identified, confirming the progenitor candidate identification based on the AO image. North is up and east is to the left in all panels.

were placed in the other bands; these are all at the $5 \sigma$ level. The detection limit in 2012 in $F 435 W$ is 26.8 . The limits in 2020 are $25.1,25.2,26.5$, and $26.8 \mathrm{mag}$ in $F 275 W, F 336 \mathrm{~W}, F 438 \mathrm{~W}$, and $F 555 W$, respectively. The lack of detection of the star in bands bluer than $F 814 W$ indicates that this is a cool, red star.

The detected star, given the assumed distance modulus and accounting only for MW extinction, has absolute magnitudes of $M_{F 814 W} \approx-5.8, M_{F 275 W}>-5.8, M_{F 336 W}>-5.7, M_{F 438 W}>$ -4.4 , and $M_{F 555 W}>-4.1$. For red supergiants (RSGs) near the terminus of their evolutionary tracks, from the BPASS solarmetallicity single-star models (Stanway \& Eldridge 2018) at $10-15 M_{\odot}$, we would expect $M_{F 814 W} \approx-7$ to $-8 \mathrm{mag}$. This implies that the progenitor may have been either more compact or further extinguished by $A_{F 814 W} \gtrsim 1 \mathrm{mag}$, potentially from circumstellar dust.
We furthermore obtained additional image data in $F 555 \mathrm{~W}$ and $F 814 W$ on 2021 July 28 with the HST WFC3/UVIS ${ }^{14}$. The $\mathrm{SN}$ is still prominent in the images (Fig. 3, bottom right), and we confirm, with an astrometric $1 \sigma$ uncertainty of 0.15 UVIS pixels (6 milliarcsec), the identification of the progenitor candidate, based on the AO image.

\subsubsection{Precursor study}

The ZTF survey first started to monitor the position where SN 2020jfo exploded more than 800 days $(2.4 \mathrm{yr}$ ) before the explosion date. We obtained in total 300 pre-explosion observations during 158 different nights. No precursors were detected

${ }^{14}$ GO-16179; PI: A. Filippenko. 

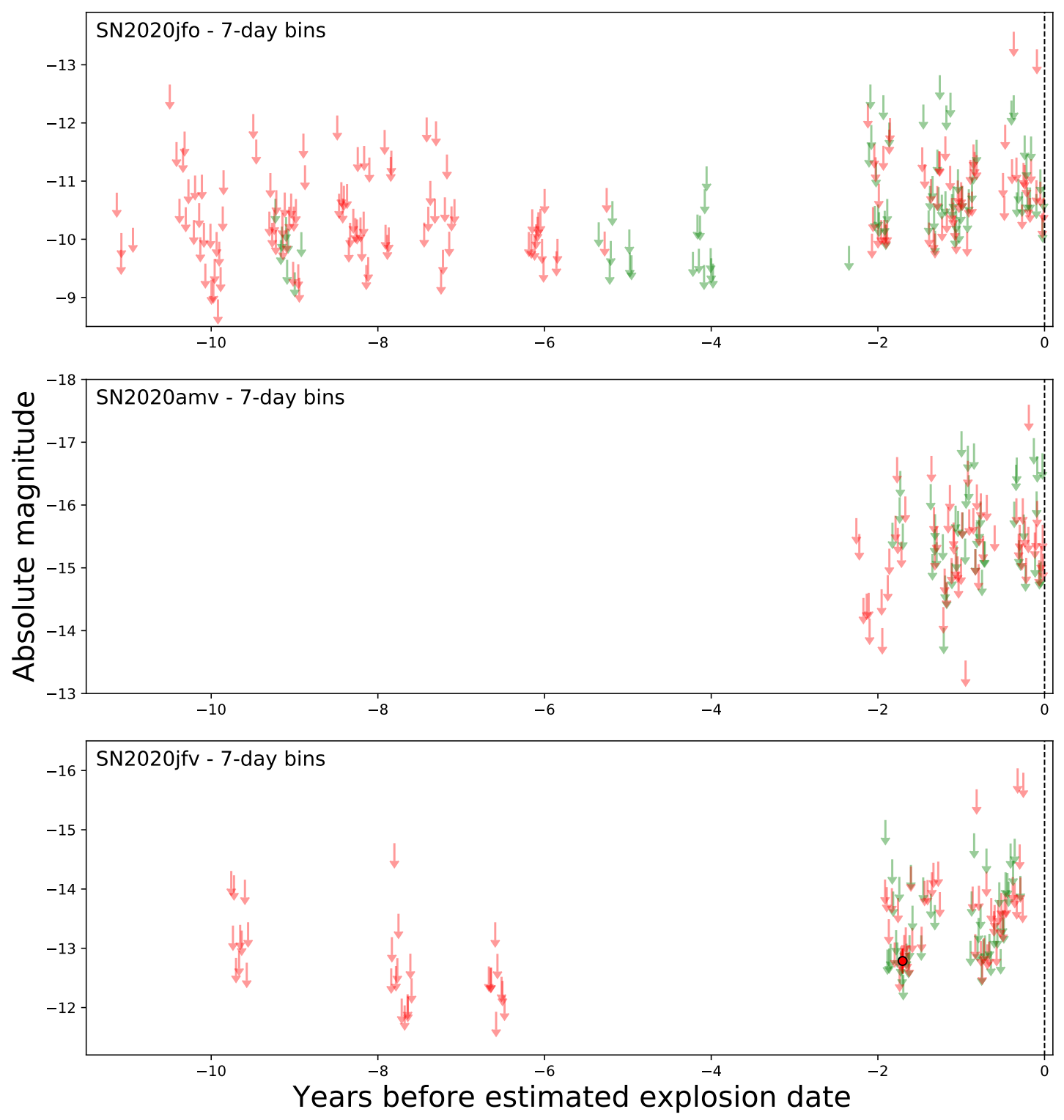

Fig. 4. Pre-explosion light curves from both (i)PTF and ZTF of the three SNe in 7-day bins. No firm detections were obtained in the $11 \mathrm{yr}$ prior to explosion, and the limits are discussed in the text.

when searching the unbinned or binned data following the methods described by Strotjohann et al. (2021). The median limiting magnitude is -11 in the $r$ band and brighter precursors can be excluded in 31 weeks or $25 \%$ of the time, while outbursts as bright as magnitude -12 can be ruled out $49 \%$ of the time within the last $2.4 \mathrm{yr}$ before the SN explosion. The SN location was also regularly observed by the Palomar Transient Factory (PTF) and the intermediate PTF (iPTF) surveys; 988 observations were obtained spanning 11.1 to $4 \mathrm{yr}$ before the $\mathrm{SN}$ explosion. We can rule out week-long precursors that are brighter than magnitude -11 in the Mould $R$ band in 102 weeks or $28 \%$ of the time. The upper panel of Fig. 4 shows the limiting magnitudes for weeklong bins.

A similar precursor search for SN 2020amv also did not reveal any pre-explosion activity, as shown in the middle panel of Fig. 4, but the limits are less constraining owing to the larger SN distance. The median limiting magnitude is -15.7 in the $r$ band and such a bright outburst can be excluded in 30 weeks or $25 \%$ of the time. The detected flash-spectroscopy features in the early-time spectra of SN 2020amv indicate that the progenitor star lost material shortly before the explosion, but apparently this mass-loss event was not associated with any optical outburst that was bright enough to be detected in this search. This is consistent with Strotjohann et al. (2021), who observed no pre-explosion outbursts prior to $20 \mathrm{SNe}$ with flash-spectroscopy features, including SN 2020amv, even though several of them were located at small redshifts of $z<0.02$. According to Strotjohann et al. (2021), this indicates that these flash-spectroscopy SNe likely have fewer or fainter outbursts than Type IIn SNe, but their sample was too small to constrain the precursor rate further. SN 2020amv reveals, as described below, both early flash features, but also later evidence for CSM interaction.

For SN 2020jfv, a single bin surpasses the formal $5 \sigma$ threshold of the precursor search. The detection occurs $1.8 \mathrm{yr}$ before the estimated explosion date in the $r$ band and is seen when combining data in 7-day or 30-day bins. However, a more detailed inspection shows that this is likely a false detection. No point 
Table 7. Summary of spectroscopic observations.

\begin{tabular}{|c|c|c|c|}
\hline Object & $\begin{array}{l}\text { UT observation date } \\
\text { (YYYY MM DD) }\end{array}$ & $\begin{array}{l}\text { Rest-frame phase } \\
\text { (days) }\end{array}$ & Telescope+Instrument \\
\hline SN 2020jfo & 2020 May 06 & 1.21 & $\mathrm{LT}+\mathrm{SPRAT}$ \\
\hline SN 2020jfo & 2020 May 06 & 1.22 & $\mathrm{NOT}+\mathrm{ALFOSC}$ \\
\hline SN 2020jfo & 2020 May 07 & 1.66 & P60+SEDM \\
\hline SN 2020jfo & 2020 May 07 & 2.22 & $\mathrm{LT}+\mathrm{SPRAT}$ \\
\hline SN 2020jfo & 2020 May 08 & 3.22 & LT+SPRAT \\
\hline SN 2020jfo & 2020 May 09 & 3.49 & P60+SEDM \\
\hline SN 2020jfo & 2020 May 10 & 4.64 & P60+SEDM \\
\hline SN 2020jfo & 2020 May 15 & 9.56 & P60+SEDM \\
\hline SN 2020jfo & 2020 May 17 & 11.50 & Lick+Kast \\
\hline SN 2020jfo & 2020 May 25 & 19.48 & Lick+Kast \\
\hline SN 2020jfo & 2020 May 25 & 19.53 & P60+SEDM \\
\hline SN 2020jfo & 2020 May 29 & 23.44 & Lick+Kast \\
\hline SN 2020jfo & 2020 Jun. 12 & 37.31 & P60+SEDM \\
\hline SN 2020jfo & 2020 Jun. 17 & 42.36 & P60+SEDM \\
\hline SN 2020jfo & 2020 Dec. 04 & 211.63 & P60+SEDM \\
\hline SN 2020jfo & 2020 Dec. 07 & 214.40 & $\mathrm{NOT}+\mathrm{ALFOSC}$ \\
\hline SN 2020jfo & 2020 Dec. 22 & 229.63 & P60+SEDM \\
\hline SN 2020jfo & 2021 Jan. 16 & 254.17 & $\mathrm{NOT}+\mathrm{ALFOSC}$ \\
\hline SN 2020jfo & 2021 Feb. 04 & 273.33 & P60+SEDM \\
\hline SN 2020jfo & 2021 Feb. 09 & 277.93 & $\mathrm{NOT}+\mathrm{ALFOSC}$ \\
\hline SN 2020jfo & 2021 Mar. 09 & 305.77 & $\mathrm{NOT}+\mathrm{ALFOSC}$ \\
\hline SN 2020jfo & 2021 Apr. 20 & 348.41 & $\mathrm{NOT}+\mathrm{ALFOSC}$ \\
\hline SN 2020amv & 2020 Jan. 24 & 1.67 & P60+SEDM \\
\hline SN 2020amv & 2020 Jan. 28 & 5.42 & P60+SEDM \\
\hline SN 2020amv & 2020 Jan. 29 & 6.59 & GeminiN+GMOS \\
\hline SN 2020amv & 2020 Jan. 31 & 8.12 & P60+SEDM \\
\hline SN 2020amv & 2020 Feb. 02 & 9.84 & $\mathrm{NTT}+\mathrm{EFOSC} 2^{(a)}$ \\
\hline SN 2020amv & 2020 Feb. 04 & 12.03 & P60+SEDM \\
\hline SN 2020amv & 2020 Feb. 06 & 13.89 & P60+SEDM \\
\hline SN 2020amv & 2020 Sep. 19 & 230.37 & P60+SEDM \\
\hline SN 2020amv & 2020 Sep. 27 & 238.00 & P60+SEDM \\
\hline SN 2020amv & 2020 Nov. 18 & 287.67 & P200+DBSP \\
\hline SN 2020amv & 2021 Jan. 23 & 350.61 & $\mathrm{NOT}+\mathrm{ALFOSC}$ \\
\hline SN 2020amv & 2021 Feb. 14 & 372.35 & $\mathrm{NOT}+\mathrm{ALFOSC}$ \\
\hline SN 2020amv & 2021 Apr. 07 & 421.58 & Keck+LRIS \\
\hline SN 2020jfv & 2020 Jun. 20 & 50.55 & P60+SEDM \\
\hline SN 2020jfv & 2020 Jun. 24 & 54.42 & P60+SEDM \\
\hline SN 2020jfv & 2020 Dec. 01 & 211.58 & P60+SEDM \\
\hline SN 2020jfv & 2020 Dec. 06 & 217.18 & $\mathrm{NOT}+\mathrm{ALFOSC}$ \\
\hline SN 2020jfv & 2020 Dec. 07 & 217.45 & P60+SEDM \\
\hline SN 2020jfv & 2021 Jan. 14 & 254.89 & Keck+LRIS \\
\hline SN 2020jfv & 2021 Jul. 08 & 426.89 & $\mathrm{NOT}+\mathrm{ALFOSC}$ \\
\hline
\end{tabular}

Notes. ${ }^{(a)}$ Uploaded from TNS from ePESSTO.

source is seen when coadding the 3 difference images in the bin, and the $g$-band images in the same nights yield a significantly deeper limiting magnitude. The median limiting magnitude is close to -14 in the $r$ band and we can exclude such a bright precursor in 29 weeks during the final $2 \mathrm{yr}$ before the $\mathrm{SN}$ explosion ( $28 \%$ of the time), assuming that the mentioned single detected flux excess is not real. We also analyzed $158 \mathrm{PTF} / \mathrm{iPTF}$ observations and can exclude precursors brighter than magnitude -14 in 31 weeks.

\subsection{Optical spectroscopy}

Follow-up spectroscopy of SN 2020jfo was primarily conducted with robotic telescopes, most of them with the SEDM mounted on the P60, but also with SPRAT on the LT. Further spectra were obtained with the NOT using ALFOSC. This included the abovementioned classification spectrum for SN 2020jfo, but also later nebular-phase spectra. We also obtained spectra with the Lick $3 \mathrm{~m}$ Shane telescope equipped with the Kast spectrograph. The full log of spectra is provided in Table 7. As can be seen in the table, P60+SEDM was also instrumental in providing spectra for the other two SNe. For the three $\mathrm{SNe}, 42$ spectra in total where obtained. Additional spectra in this paper come from GeminiNorth equipped with GMOS, the Palomar P200 telescope and DBSP (Oke \& Gunn 1982), as well as deep nebular spectra taken with the Keck-I telescope using the Low Resolution Imaging Spectrograph (LRIS; Oke et al. 1995).

The LPipe reduction pipeline (Perley 2019) was used to process the LRIS data. SEDM spectra were reduced using the pipeline described by Rigault et al. (2019), and the spectra from La Palma were reduced using standard pipelines and procedures for each telescope and instrument. The ALFOSC spectra were often reduced using PypeIt (Prochaska et al. 2020). All spectral data and corresponding information is available via WISeREP ${ }^{15}$ (Yaron \& Gal-Yam 2012). All spectra have been calibrated on an absolute scale using contemporaneous (or interpolated) photometry.

\section{Spectropolarimetry}

We obtained two epochs of spectropolarimetry of SN 2020jfo on the nights of 2020 May 25 and May 29 (19.7 and 23.7 restframe days past explosion) using the polarimetry mode of the Kast spectrograph on the Lick $3 \mathrm{~m}$ Shane telescope. On each night, low-polarization and high-polarization standard stars were observed to calibrate the data. Observations and data reduction were carried out as in Patra et al. (in prep.).

Linear polarization is calculated from the Stokes $Q$ and $U$ parameters as $P=\sqrt{Q^{2}+U^{2}}$, and the polarization position angle (PA) on the sky is defined as $\theta=1 / 2 \arctan (U / Q)$. $P$ is a positive-definitive quantity and therefore overestimated when the signal-to-noise ratio $(\mathrm{S} / \mathrm{N})$ is low. We debias the polarization as

$P_{\mathrm{db}}=P-\left(\frac{\sigma_{\mathrm{P}}^{2}}{P} \times h\left(P-\sigma_{\mathrm{P}}\right)\right)$ and $\theta_{\mathrm{db}}=\theta$,

where $\sigma_{P}$ is the $1 \sigma$ uncertainty in $P$ and $h$ is the Heaviside step function.

We measured the average Stokes $Q$ and $U$ of the lowpolarization star HD 110897 to $<0.05 \%$, demonstrating the low instrumental polarization. We also find low interstellar polarization (ISP) in the direction of SN 2020jfo. Serkowski et al. (1975) showed that an upper limit on ISP owing to dichroic extinction by dust grains can be derived as $9 \times E(B-V)$. In the direction of SN 2020jfo, the estimated $E(B-V)=0.02$ mag implies that ISP $<0.18 \%$. We confirm that the Galactic ISP is low by measuring the polarization of an ISP "probe-star" close to the line of sight to SN 2020jfo. We found the polarization of the probe star to be $<0.15 \%$. Furthermore, the emission peak of the $\mathrm{H} \alpha$ feature of a $\mathrm{SN}$ is expected to be depolarized due to contamination by unpolarized flux diluting the underlying polarized continuum flux. The minimum observed polarization in the $\mathrm{H} \alpha$ feature also constrains the ISP to be $<0.2 \%$. Taken together, the three lines of evidence all show that the ISP in the direction of SN 2020jfo is low.

The continuum polarization, which reflects the global ejecta asymmetry, is $0.4-0.7 \%$ over the two epochs, typical of SNe II while on the plateau (see Wang \& Wheeler 2008, and references therein).

\footnotetext{
15 https://wiserep.weizmann.ac.il

16 We observed the star Gaia ID 3894181087039808128.
} 


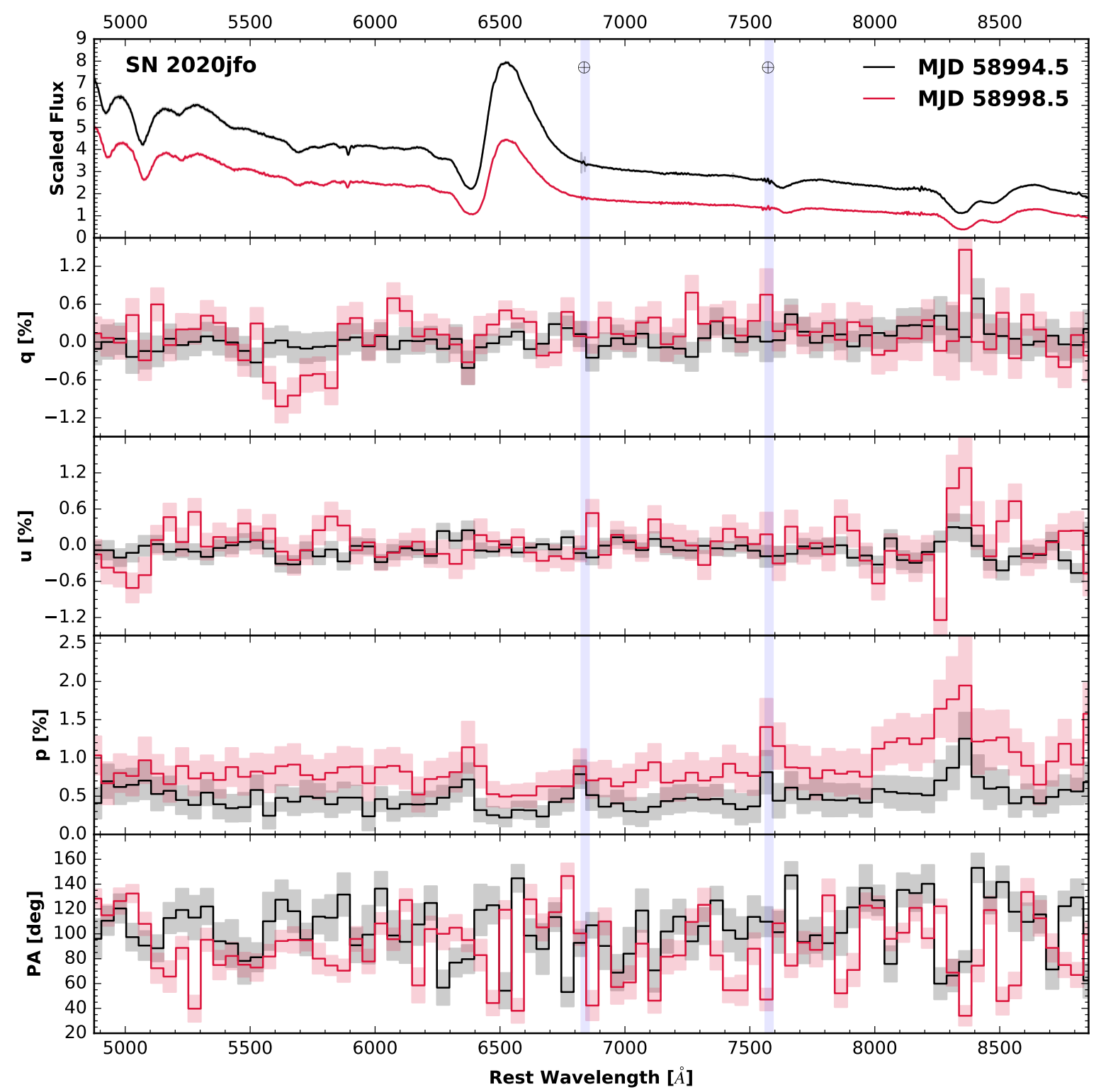

Fig. 5. Spectropolarimetry of SN 2020jfo on 2020 May 25 and May 29. The panels (from top to bottom) show total flux, Stokes $Q$, Stokes $U$, debiased polarization, and the position angle. The vertical bands represent the regions of potential telluric overcorrection. The data, except for total flux, are binned to $50 \AA$ to improve the $\mathrm{S} / \mathrm{N}$.

The polarization position angle hovers around a mean of $\sim 100^{\circ}$ over the two epochs. The lack of significant change in PA could imply a global axis of symmetry, although the data do not have sufficiently long temporal coverage to confirm this. The Ca II NIR feature is significantly polarized, with levels exceeding $1 \%$ at both epochs (see Fig. 5). The high line polarization suggests that $\mathrm{Ca}$ is not uniformly distributed within the ejecta and likely exists in clumps. The Ca II line polarization also shows velocity-dependent variation, with the high-velocity feature more polarized than the normal-velocity feature.

\section{Results and discussion}

\subsection{Light curves}

The LCs of our three SNe are displayed in Fig. 2. We first focus on the LC of SN 2020jfo.

\subsubsection{SN 2020jfo}

As mentioned above, we have fairly good constraints on the explosion epoch with an uncertainty of \pm 2 days simply based on the last nondetection. Therefore, we can also measure the rise time with some precision. We used a Gaussian Processing (GP) algorithm ${ }^{17}$ to interpolate the photometric data and measure the rise time and the peak magnitudes. The results are provided in Table 8 . The $\mathrm{SN}$ rises to maximum brightness in slightly less than 5 days in both $g$ and $r$. The peak magnitude of $m_{r}^{\text {peak }}=14.4$ made SN 2020jfo one of the brighter CC SNe during 2020.

After the initial rise follows a plateau phase in the $r$ band of $\sim 60+$ days, which establish SN 2020jfo as a Type IIP SN. The $i$ band is well sampled the first 40+ days, when it follows the plateau, whereas the $g$ band declines faster. The LCs cover the first 60 days well, whereafter the SN position was too close to the Sun in the sky. It was recovered after solar conjunction

17 https://george. readthedocs. io with a Matern32 kernel. 
Table 8. Supernova light-curve properties.

\begin{tabular}{lcccccccccc}
\hline \hline SN name & $t_{g}^{\text {rise }}$ & $\begin{array}{c}t_{r}^{\text {rise }} \\
\text { (rest-frame days past explosion) }\end{array}$ & $t_{i}^{\text {rise }}$ & $m_{g}^{\text {peak }}$ & $\begin{array}{l}m_{r}^{\text {peak }} \\
(\mathrm{mag})\end{array}$ & $m_{i}^{\text {peak }}$ & $M_{g}^{\text {peak }}$ & $\begin{array}{c}M_{r}^{\text {peak }} \\
(\mathrm{mag})\end{array}$ & $M_{i}^{\text {peak }}$ \\
\hline SN 2020amv & 8.99 & 14.16 & 16.46 & 17.38 & 17.57 & 17.62 & -19.23 & -19.04 & -18.99 \\
SN 2020jfo & 4.68 & 4.87 & 5.97 & 14.38 & 14.37 & 14.65 & -16.49 & -16.50 & -16.22 \\
SN 2020jfv & - & - & - & $<18.53$ & 17.71 & 17.28 & -15.95 & -16.77 & -17.22 \\
\hline
\end{tabular}

in $g$ and $r$, declining linearly up to $\sim 350$ days. The forcedphotometry ATLAS LCs confirm the Type IIP classification and the general shape of the LC. In fact, the fall off the plateau is best seen in the $o$ band, which indicates a plateau length of $64 \pm 3$ days $^{18}$. In comparison with the large SN II sample of Anderson et al. (2014), this is actually one of the shortest plateau lengths. We followed the SN up to a year after explosion.

The color evolution in $g-r$ for SN 2020jfo and also for the Type II SN $2020 \mathrm{amv}$ initially become redder for the first 60 days, while SN 2020jfo was on the plateau. At about 200 days past explosion all our three SNe display $g-r \approx 1.0$ mag. SN 2013ej (Valenti et al. 2014; Yuan et al. 2016), which is known to experience little host-galaxy extinction, show a similar initial reddening. Since our three SNe are bluer than SN 2013ej, this at least conforms with our omission of extra host extinction corrections.

In Fig. 6 we show the LCs in absolute magnitudes $\left(M_{r}\right)$ together with the LCs of a few other SNe II. The magnitudes in Fig. 6 are in the AB system ${ }^{19}$ and have been corrected for distance modulus, MW extinction, and host extinction if any, and are plotted versus rest-frame days past estimated explosion epoch. SN 2020jfo reached a peak magnitude quite similar to that of the canonical Type IIP SN $1999 \mathrm{em}^{20}$, but the plateau phase is significantly shorter. At nebular phases SN 2020jfo is fainter than SN $1999 \mathrm{em}$, but instead follows the same decline rate and tail luminosity as the Type II SN 2013ej ${ }^{21}$.

In order to estimate the total radiative output, we also attempted to construct bolometric LCs. For SN 2020jfo, we adopted two approaches: we use a black-body (BB) function fitted to the GP interpolated fluxes, and we also employ an analytic bolometric correction (BC) that was constructed by Lyman et al. (2016) from a sample of SNe II. On the LC plateau, where we have coverage from the NIR to the UV, we can construct spectral energy distributions (SEDs) and fit to a diluted BB function. Integrating that $\mathrm{BB}$ function provides the bolometric luminosity. Comparing this with the prescription from Lyman et al. (2016), we find that the bolometric LC on the plateau agrees very well with our BB estimate (the ratio is $0.97 \pm 0.05$ on the late plateau). Therefore, for the part of the LC where we only have optical data, we follow the Lyman et al. (2016) method. The only difference between these methods is for the very early phases, where our UV data imply slightly higher luminosities; this is illustrated in Fig. 7. The bolometric LC is also provided on WISEREP.

Using this, we can estimate a maximum bolometric luminosity for SN 2020jfo of $L_{\text {bol }}=1.63 \times 10^{42} \mathrm{erg} \mathrm{s}^{-1}$ at 4 rest-frame

\footnotetext{
18 As done by Anderson et al. (2014), we fit $o$-band data using a $\chi^{2}$ minimizing procedure with a composite function of a Gaussian, FermiDirac, and a straight line, following Felipe Olivares et al. (2010).

19 The Vega/AB magnitude conversion follows Blanton \& Roweis (2007).

${ }^{20}$ We used $E(B-V)=0.035 \mathrm{mag}$ and a distance of $7.5 \mathrm{Mpc}$ from Hamuy et al. (2001) for SN 1999em, data from Faran et al. (2014).

${ }^{21}$ We used $E(B-V)=0.060 \mathrm{mag}$ and a distance of $9.1 \mathrm{Mpc}$ from Valenti et al. (2014), Yuan et al. (2016) for SN 2013ej.
}

days and a total radiated energy over the first 350 rest-frame days of $E_{\mathrm{rad}}=5.74 \times 10^{48} \mathrm{erg}$. The radioactive ${ }^{56} \mathrm{Ni}$ mass ejected in the explosion can be inferred by measuring the luminosity tail, which is powered by the decay of radioactive ${ }^{56} \mathrm{Co}$. Using $L=1.45 \times 10^{43} \exp \left(-t / \tau_{\mathrm{Co}}\right)\left(M_{\mathrm{Ni}} / M_{\odot}\right) \mathrm{erg} \mathrm{s}^{-1}$ from Nadyozhin (2003) implies that we would require $0.024 \pm 0.002 M_{\odot}$ of ${ }^{56} \mathrm{Ni}$ to account for the luminosity (70-100 rest-frame days; see further Sect. 3.1.3).

\subsubsection{Comparisons with SNe 2020amv and 2020jfv}

We here discuss the LCs of the other two SNe presented in this paper, although not at the same level of detail as for SN 2020jfo. SN 2020amv was photometrically monitored with P48 for more than a year, with a few data points also provided by LT, P60, and NOT. The LC is displayed in the middle panel of Fig. 2. The explosion date is well constrained and the $\mathrm{SN}$ rose in a bit more than two weeks ( $r$ band; Table 8 ) to a Gaussian-shaped LC peak, where both the rise and the fall are somewhat faster in $g$ than in $r$, with $\Delta m_{15}^{r}=0.45 \pm 0.02 \mathrm{mag}$ and $\Delta m_{15}^{g}=0.52 \pm 0.01 \mathrm{mag}$. After this initial phase, the LC is rejuvenated, as the $r$-band brightness gently rises again some 60 days past peak. Overall, it is a very long-lived SN which we followed for more than 450 days. The absolute-magnitude LC (Fig. 6) demonstrates that SN 2020amv was very luminous, $M_{g}^{\text {peak }}=-19.2 \mathrm{mag}$, the initial peak resembling that of a Type I SN, but the remaining bumpy, bright, and long-lived LC reveals that the (late-time) power source must be something in addition to radioactive decay. In terms of CC SNe, such LCs are expected to be powered by CSM interaction (e.g., Nyholm et al. 2017, 2020), but we note that superluminous SNe, even of Type I, sometimes display long-lived bumpy LCs, which are not always easily explained in terms of a central engine (e.g., the large SLSN-I sample from ZTF; Chen et al., in prep.).

SN 2020jfv was found while declining and we do not have good constraints for the date of explosion or the early peak. The right-hand panel of Fig. 2 therefore only shows a declining and eventually flattening LC. The unusual aspect is that the $r$-band LC, after declining a full magnitude over the first $\sim 100$ days, rises by $\sim 0.3$ mag in the next 100 days, before the target was lost in the Sun's glare. After solar conjunction, we recovered the LC in gri at virtually the same magnitudes as before the gap.

\subsubsection{Light-curve modeling for SN2020jfo}

In order to estimate progenitor and explosion parameters for SN2020jfo from the bolometric LC, we make use of the semi-analytic Monte Carlo code that was recently presented by Jäger et al. (2020), as used for the low-luminosity Type IIP SN 2020cxd (Yang et al. 2021). After marginalization, our fit provides estimates with confidence intervals $(2 \sigma)$ for each of the parameters: SN 2020jfo has $M_{\mathrm{ej}}=5.16_{-2.00}^{+0.28} M_{\odot}$, $E_{\text {kin }}=2.04_{-1.02}^{+0.57} \times 10^{51} \mathrm{erg}$, and $v_{\exp }=8.14_{-1.32}^{+0.54} \times 10^{3} \mathrm{~km} \mathrm{~s}^{-1}$ for the ejecta mass, kinetic energy, and expansion velocity 


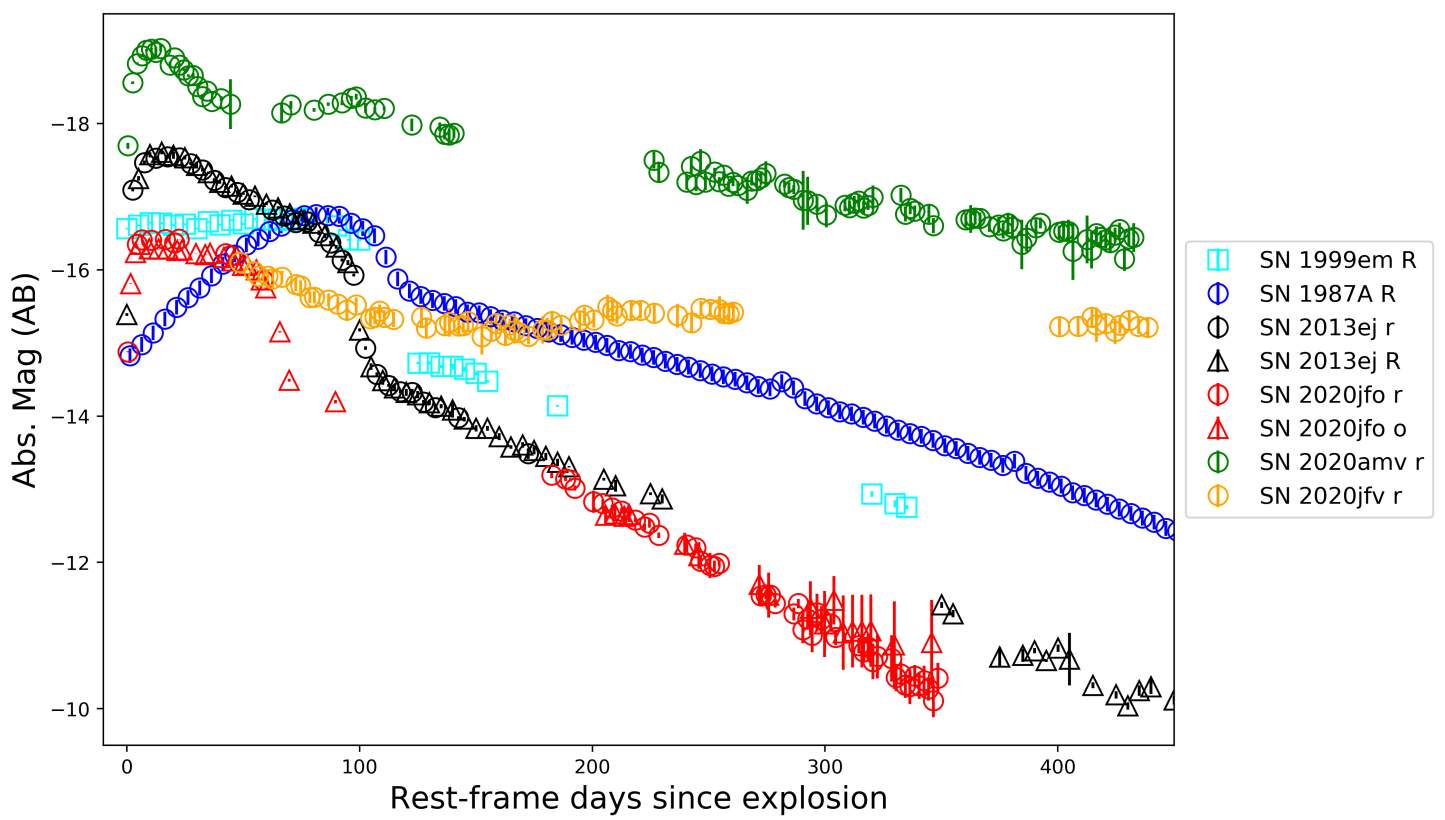

Fig. 6. Light curves in absolute magnitudes $\left(M_{r}\right)$ for our three SNe. This accounts for distance modulus and MW extinction as discussed in the text, but no additional corrections for host extinction. The comparison SNe are introduced in the text. The photometry has been binned to nightly averages.

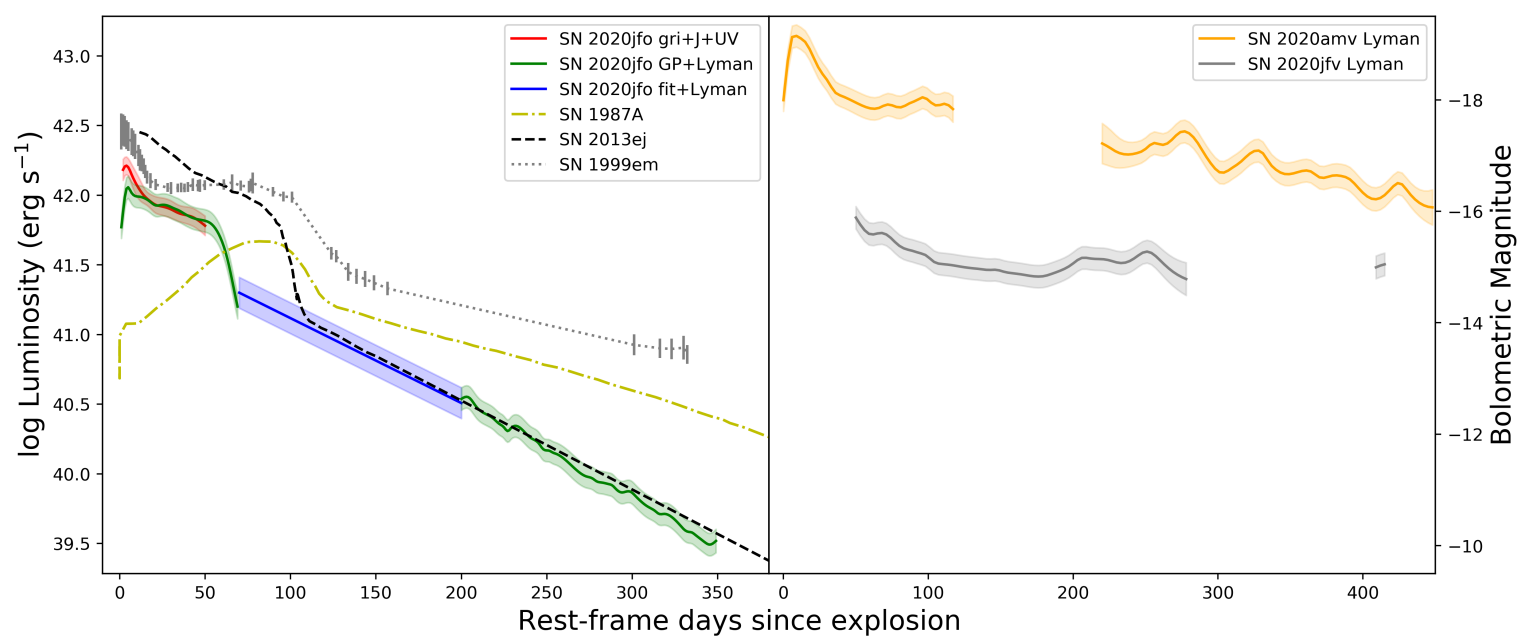

Fig. 7. Bolometric luminosities for our SNe. Left: SN 2020jfo with some comparison objects (see text). The green solid lines with shaded regions are estimated with the Lyman et al. (2016) method and is a Gaussian Process fit to that LC. The blue region is a linear interpolation for the region with less data. The red fit on the plateau is for a diluted BB fit to our UV through NIR data, and matches the Lyman et al. method well on the later part of the plateau. Right: SNe 2020amv and 2020jfv. These were derived using the Lyman et al. method, which is rather approximate given the unusual nature of these $\mathrm{SNe}$.

(respectively). The nickel mass was simultaneously estimated as $0.029 \pm 0.014 M_{\odot}$. The mass of radioactive nickel is thus similar to that estimated for SN 2013ej $\left(0.023 M_{\odot}\right.$; Yuan et al. 2016), as expected from the similar absolute magnitudes (Fig. 6). It also matches the estimate from Sect. 3.1.1. Moreover, the LC slope is similar for these two SNe, and Yuan et al. (2016) interpreted this as being due to gamma-ray escape. The same seems to apply here. In fact, we can fit for a gamma-leakage LC (Sollerman et al. 1998) with the flux declining as $e^{(-t / 111.3)} \times(1-$ $\left.0.965 \times e^{-\left(t_{0} / t\right)^{2}}\right)$, where $t$ is the time in days and $t_{0}$ is the epoch when the optical depth to the gamma rays is unity. This epoch is also related to the ejecta mass (Clocchiatti \& Wheeler 1997, their Eq. (5)), and our best-fit values $M_{\mathrm{Ni}}=0.025 M_{\odot}$ and $t_{0}=$ 166 days correspond to $M_{\mathrm{ej}} \approx 5-6 M_{\odot}$, for $(1-1.7) \times 10^{51} \mathrm{erg}$ of kinetic energy. We note that the estimated ejecta mass is low, in agreement with the results from the Monte Carlo fit to the short plateau.

In this respect, the short plateau of SN 2020jfo is similar to those discussed by Hiramatsu et al. (2021). They presented three Type IIP SNe having plateau lengths of only 50-70 days, arguing that this was due to low ejecta mass. They further suggested that their $\mathrm{SNe}$ originated from massive progenitors with normal to large amounts of radioactive nickel, and that the required large mass loss was also likely the cause for the bright early luminosity. For SN 2020jfo we have a normal initial luminosity and a modest amount of radioactive nickel. There are no signs of CSM interaction from either the LC or the spectra. In addition, we have evidence that the initial mass was not very large (Sect. 3.3). 


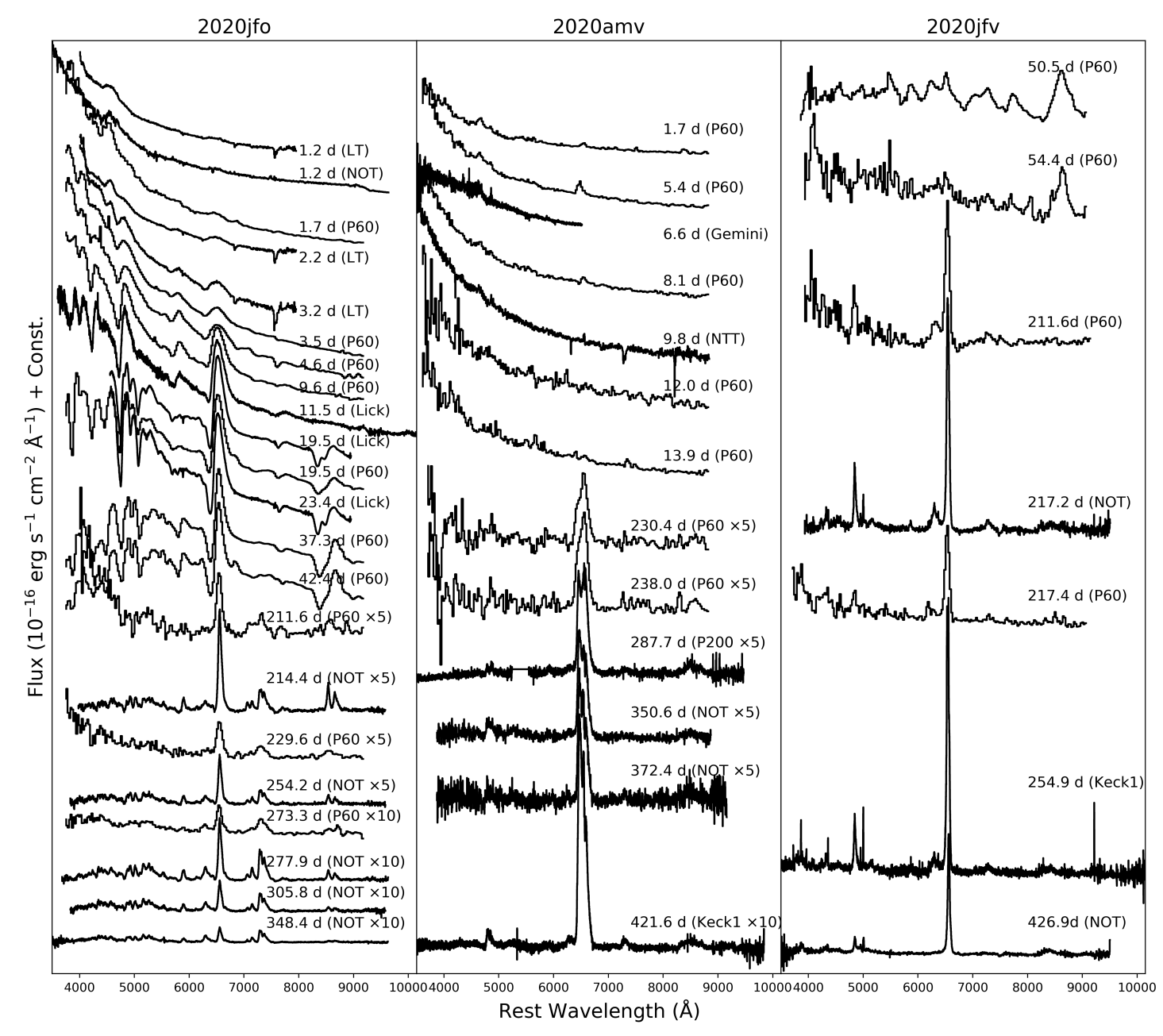

Fig. 8. Sequence of spectra of SNe 2020jfo, 2020amv, and 2020jfv. The epochs and scale factors of the spectra are also provided.

SN 2020jfo is therefore somewhat different from the discussion of Hiramatsu et al. (2021) in the sense that the plateau is not generally powered by the radioactivity.

\subsection{Spectroscopy}

The spectroscopic sequence for SN 2020jfo, as provided in Table 7, is displayed in Fig. 8. The photospheric phase is well covered by the robotic telescopes, and although the resolution of the SEDM spectra is low, the overall spectral evolution as the photosphere expands and cools is nicely covered. The early classification spectra show shallow Balmer lines and a He II feature at $15000 \mathrm{~km} \mathrm{~s}^{-1}$. No signatures of CSM interaction are present in the spectra. A total of 22 spectra are presented for SN 2020jfo, covering phases from 2 to 350 days past explosion. The photospheric velocities, as estimated from the P Cygni Balmer lines, is $\sim 9000 \mathrm{~km} \mathrm{~s}^{-1}$ at the early plateau and $\sim 7000 \mathrm{~km} \mathrm{~s}^{-1}$ toward the end of the plateau (44 days past explosion).

\subsubsection{SN 2020amv}

Thirteen spectra were obtained of SN 2020amv (Table 7). The first spectrum was acquired with the SEDM about 1.7 days from the estimated explosion date. Despite the low resolution, several narrow emission lines are identified (e.g., $\mathrm{H} \alpha, \mathrm{H} \beta$ ), including those of highly ionized species (He II 24686) which correspond to flash-ionization lines (Bruch et al. 2021). These features dis- appear within 13 days from the explosion epoch. This is thus one of the more long-lived flash features in the ZTF sample (Bruch et al, in prep.). Such transient emission lines emerge from the early interaction of the shock-breakout radiation with a nearby (typically $\lesssim 10^{15} \mathrm{~cm}$; e.g., Yaron et al. 2017) CSM. Two higher-resolution spectra were obtained at 6.6 and 9.8 days. The narrow $\mathrm{H} \beta$ emission line in the former indicates a relatively slow velocity of $\lesssim 335 \mathrm{~km} \mathrm{~s}^{-1}$ (full width at half-maximum intensity; FWHM), consistent with a wind velocity of the CSM. The following spectra show mainly a blue continuum. After the rebrightening of the light curve was recognized, additional spectra were obtained with SEDM 230 days past explosion. These showed a strong $\mathrm{H} \alpha$ line, with very high velocities. This triggered us to obtain a higher-resolution spectrum with the P200 (Table 7), revealing a broad, boxy line profile having several emission peaks. This complex line profile slowly evolves in later spectra, and the nebular lines are further explored in Sect. 3.5.

SN 2020amv can in some sense also be seen as a transforming SN (as we argue for SN 2020jfv below). It seems that CSM interaction is the driving force in powering SN 2020amv, but that the evidence for this is manifested in different ways throughout the SN evolution. The early flash spectroscopy provides evidence for dense CSM close to the exploding star, although the early LC is similar to Gaussian-shaped LCs of other types of stripped-envelope SNe. Other studies have found that such LCs might still be consistent with radioactive powering, as in SN 2018ijp (Tartaglia et al. 2021), or SN 2020eyj (Kool et al., in 


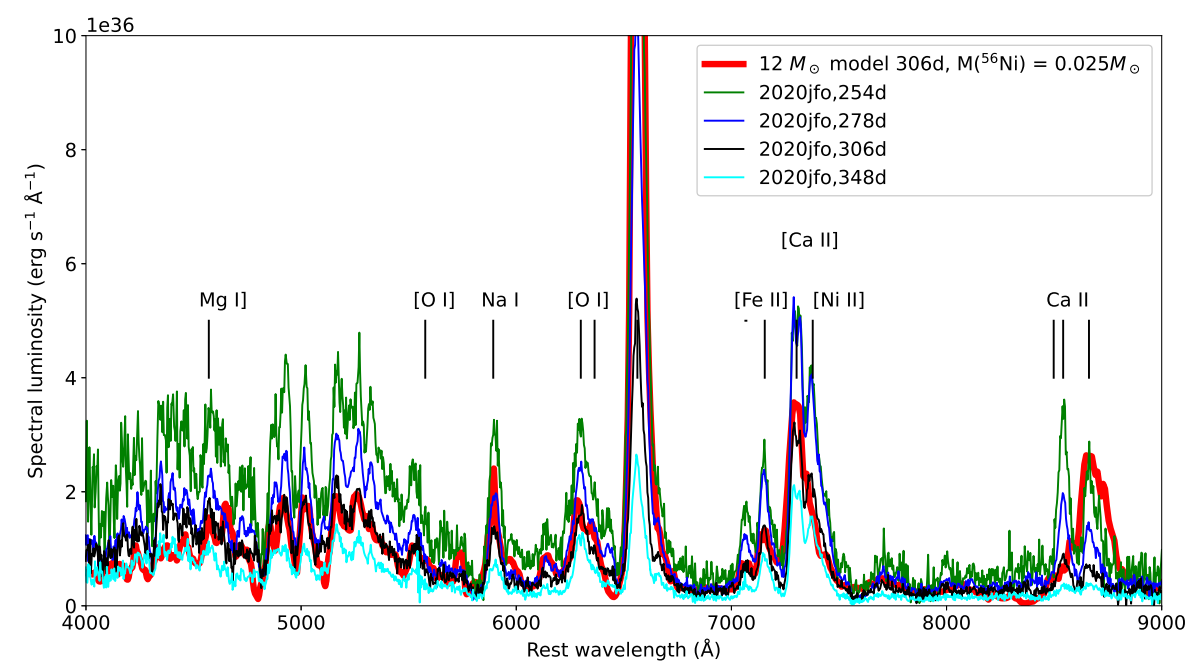

Fig. 9. Four nebular spectra from NOT for SN 2020jfo are compared with the nebular model for a $12 M_{\odot}$ explosion. The model is scaled by the ratio of ${ }^{56} \mathrm{Ni}$ masses in SN 2020jfo $\left(0.025 M_{\odot}\right)$ and the model $\left(0.062 M_{\odot}\right)$, as well as a factor of 0.5 to account for a significantly lower degree of gamma-ray trapping in SN 2020jfo. The most conspicuous emission lines are marked: [Mg I] $\lambda 4571$, [O I] $\lambda 5577$, [O I] $\lambda \lambda 6300,6364, \mathrm{Na}$ I D, H $\alpha$, [Fe II] $\lambda 7155$, [Ni II] $\lambda 7378$, [Ca II] $\lambda \lambda 7291,7323$, and the Ca II NIR triplet.

prep.), where CSM interaction came in later to power the prolonged LCs. SN 2020amv peaks at $-19.24 \mathrm{mag}$ in $g$, similar to the peak luminosity of SNe Ia. The CSM evidence in terms of LC evolution is instead obvious after about 50 days, when the second peak anticipates the long-lived nature of the overall LC. Here we lack spectroscopy, but when the spectroscopic campaign resumed it revealed nebular box-shaped emission lines (Sect. 3.5) that are a telltale signature for CSM interaction - this time the signature unfolds a cold dense shell likely caused by the reverse shock produced by ejecta running into the CSM. These are all different manifestations of CSM interaction that together unveil the mass-loss history of the exploded star.

\subsubsection{SN 2020jfv}

SN 2020jfv was first spectroscopically observed with the SEDM on P60, and based on this spectrum classified as a Type IIb SN (Dahiwale \& Fremling 2020b). Helium lines at a velocity of $7000 \mathrm{~km} \mathrm{~s}^{-1}$ are detected. Six months later, after recognizing that the target was rebrightening again, we obtained another SEDM spectrum that seemed to be dominated by bright, narrow Balmer emission lines, typical for CSM-driven SNe IIn. We also acquired some spectra with larger telescopes, namely with the NOT and Keck (Fig. 8). These show a Type II SN spectrum containing lines from elements such as $\mathrm{Mg}, \mathrm{Ca}$, and $\mathrm{O}$ (as are typically seen in $\mathrm{CC} \mathrm{SNe}$ ), but which is very much dominated by the intermediate-width Balmer lines. We discuss the nebular spectra in the next sections.

\subsection{Modeling the oxygen mass of SN2020jfo}

In Fig. 9 we zoom in on the four high-quality late-time spectra of SN 2020jfo taken with the NOT. These were obtained over a period when the SN was 250-350 days old, and the spectroscopic evolution over that time range is very slow. Overall, it is a textbook example of a normal SN II spectrum (Jerkstrand 2017), dominated by Balmer lines that still show P Cygni absorption components, but also strong emission lines of calcium and oxygen. The spectra are indeed similar to those seen in many other nebular CC SNe, like the famous SN 1987A or the well-studied SN 2012aw.
For SN 2020jfo, where we have no evidence of CSM interaction, we can compare the nebular emission-line luminosities with modeling to estimate the oxygen mass and thus the zero-age main sequence (ZAMS) mass of the star that exploded. We use the models and methodology advanced by Jerkstrand et al. (2012, 2015a, 2018). The spectra are calibrated on an absolute scale using the photometry and corrected for extinction. Figure 9 shows the $12 M_{\odot}$ model from the work of Jerkstrand et al. (2015b). In these comparisons, we have rescaled the model flux with the ratio of ${ }^{56} \mathrm{Ni}$ mass inferred for SN 2020jfo $\left(0.025 M_{\odot}\right.$; see Sects. 3.1.1 and 3.1.3) to the model ${ }^{56} \mathrm{Ni}$ mass $\left(0.062 M_{\odot}\right)$. We have in addition multiplied the model by a factor of 0.5 to account for the significantly earlier gamma-ray escape occurring in SN 2020jfo.

The $12 M_{\odot}$ model makes the best fit to the key emission lines that diagnose the progenitor mass (oxygen, sodium, and magnesium), and suggests that the best ZAMS-mass match for SN 2020jfo is in the range $10-15 M_{\odot}$. H $\alpha$ is too strong in the model, which is consistent with an unusually low hydrogen envelope mass in this SN, likely also causing the short plateau. Having a relatively low-mass He core progenitor that has lost a large part of its $\mathrm{H}$ envelope would be most naturally consistent with binary mass loss.

However, it is also noteworthy that the metal emission lines do not appear broader than in the model. With a low envelope mass, one would expect a low He core mass and a quite high explosion energy, as inferred in Sect. 3.1.3, so the metals should have an unusually high expansion velocity. Thus, no fully selfconsistent scenario is established.

Finally, we note that the Ca II NIR triplet mismatch is a wellknown shortcoming of the models. The models give scattering of Ca II $\lambda \lambda 8498,8542$ into the Ca II $\lambda 8662$ line - but in most SNe II this is not observed to happen.

\subsection{Stable nickel in SN2020jfo}

The nebular spectra also show clear evidence for stable nickel in the form of the [Ni II] $\lambda 7378$ line (Fig. 10). This line is not always seen in SNe II; a clearly observed line typically requires an unusually weak [Ca II] doublet near $7300 \AA$ combined with an intrinsically strong nickel line. Here, it is plausible that the 


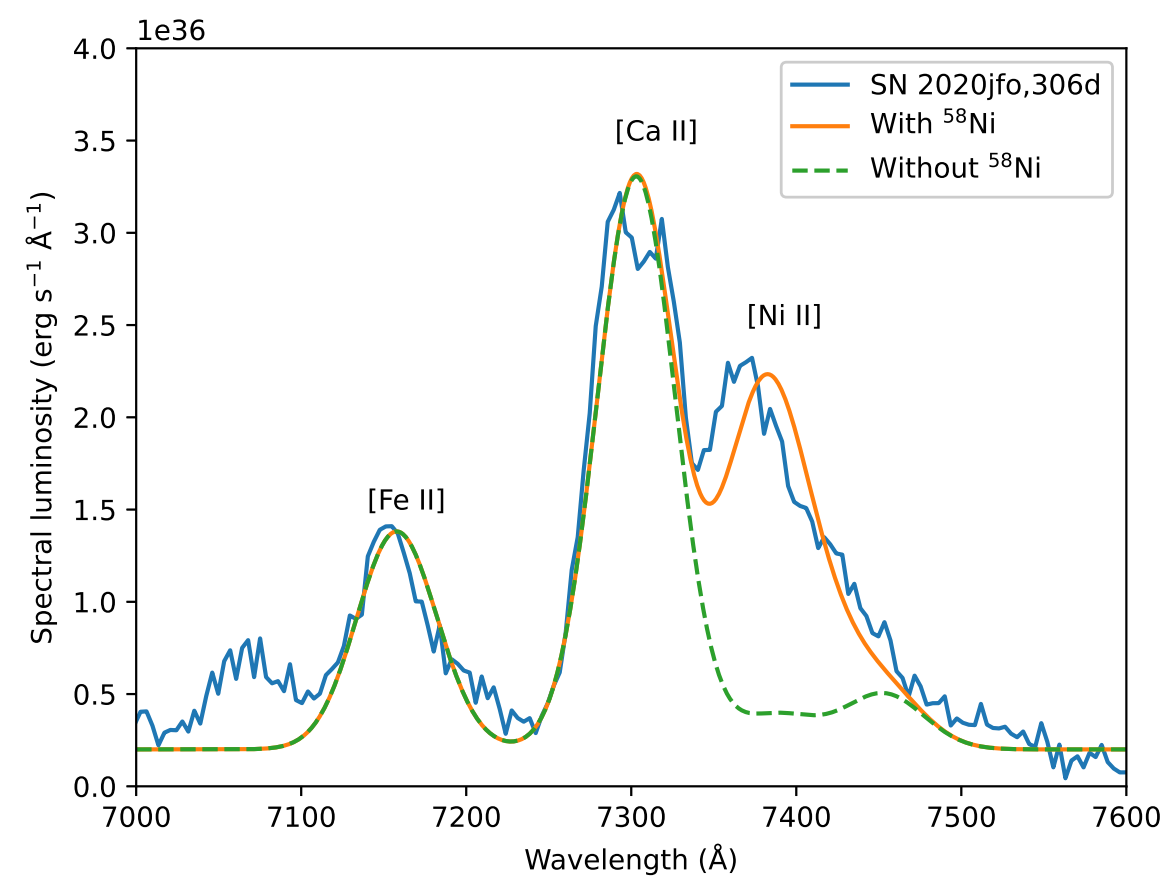

Fig. 10. Spectrum (7000-7600 ̊) for SN 2020jfo at +306 days (blue lines). It has been demonstrated that this region is dominated by emission from [Fe II], [Ni II], and [Ca II] (Jerkstrand et al. $2015 b$ ). Shown in orange is the best-fit multipleGaussian model to the emission lines following the method of Jerkstrand et al. (2015b). This fit gives $L_{\mathrm{Ni} \text { II 7378 }} / L_{\mathrm{Fe} \text { II } 7155}=1.7$, which maps to a mass ratio $M(\mathrm{Ni}) / M(\mathrm{Fe}) \approx 2$ times solar. The green dashed line shows the fit without contributions from stable nickel. small hydrogen zone damps the calcium doublet, as much of that emission comes from this zone (Li \& McCray 1993).

Stable nickel is an important diagnostic of the explosion mechanism. It is mainly composed of ${ }^{58} \mathrm{Ni}$, with a higher production most naturally being explained by burning and ejection of deeper-lying, more neutron-rich layers in the progenitor star (Jerkstrand et al. 2015b). The iron comes from decayed ${ }^{56} \mathrm{Ni}$, which with its zero neutron excess has no such dependency.

We measured the relative luminosities for the lines following the approach of Jerkstrand et al. (2015c, their Sect. 3.1.1), with a simultaneous fit to several lines predicted from the models. Even if the exact line shape is not perfectly matched, we are confident about the line identifications. The measured luminosity ratio at +306 days is $L_{\mathrm{Ni} \text { II } 7378} / L_{\mathrm{Fe} \text { II } 7155}=1.7$. The link between luminosity ratio and mass ratio depends on temperature, although quite weakly. The temperature can be estimated from the intrinsic line luminosity of [Fe II] 27155 , the best-fitting value for $M(\mathrm{Fe})=0.025 M_{\odot}$ being $2700 \mathrm{~K}$. A range of $T=2500-3000 \mathrm{~K}$ gives a mass ratio $M(\mathrm{Ni}) / M(\mathrm{Fe})=1.7-2.1$, following the analysis method of Jerkstrand et al. (2015b).

\subsection{The nebular $\mathrm{H} \alpha$ emission-line profiles}

If we focus on the strongest nebular emission line, $\mathrm{H} \alpha$, we can see that it is relatively symmetric in SN2020jfo, with $F W H M \approx 2510 \mathrm{~km} \mathrm{~s}^{-1}$. The $\mathrm{P}$ Cygni absorption has a maximum at $4000 \mathrm{~km} \mathrm{~s}^{-1}$, and also the red side of the emission line reaches that velocity. The maximum velocity in the red absorption component is $6000 \mathrm{~km} \mathrm{~s}^{-1}$, and this is actually consistent with the red shoulder of the emission line. Since we were able to properly model the nebular spectrum of SN 2020jfo in Sect. 3.3 without any input from CSM interaction, we adopt the $\mathrm{H} \alpha$ profile for this SN as a benchmark with which to compare our other two SNe, to highlight how CSM interaction (as evident in the LC evolution) can manifest itself in the line profiles.

SN 2020amv is also a SN II, but the nebular spectrum is strikingly different. We acquired two SEDM spectra at an age of 240 days, motivated by the endurance of the SN LC. The SEDM spectra revealed little more than the $\mathrm{H} \alpha$ emission line, but it caught our attention since it was very broad. The resolution of SEDM is low, but the width of the emission line stood out; we estimated a FWZI (full width at zero intensity) of $\sim 17000 \mathrm{~km} \mathrm{~s}^{-1}$. This prompted four more nebular spectra with larger telescopes. The first of these, from P200, is spectacular. It shows mainly $\mathrm{H} \alpha$, but also $\mathrm{H} \beta$ with the same line profile as well as the $\mathrm{Ca}$ II NIR triplet. The $\mathrm{H} \alpha$ line profile displays three distinct peaks, similar to the late-time spectra of SN 1998S (Pozzo et al. 2004, their Fig. 5) and SN 1993J (see e.g., Matheson et al. 2000, their Fig. 11). In Fig. 11 we show the four last nebular spectra of SN 2020amv, compared to those of other $\mathrm{SNe}$. The $\mathrm{H} \alpha$ line in the P200 spectrum has $F W H M \approx 9800 \mathrm{~km} \mathrm{~s}^{-1}$ (not measured with a Gaussian fit; FWZI $\approx 14000 \mathrm{~km} \mathrm{~s}^{-1}$ ). For the similar SN 1993J, the interpretation for the shape of the line profile was CSM interaction with emission originating in a dense thin shell, and this obviously also applies to SN 2020amv.

The P200 H $\alpha$ line profile is asymmetric. The structure on top of the rectangular, boxy, and flat-topped line profile predicted from a thin shell can be interpreted either as evidence for an overall asymmetric geometrical configuration such as a ring-like structure (as mentioned by Pozzo et al. 2004), or alternatively seen as small-scale structure as due to clumps, as suggested for the forbidden oxygen lines in SN 1993J by Spyromilio (1994). In the P200 spectrum (Fig. 11) the blue-horn emission is shifted by $5000 \mathrm{~km} \mathrm{~s}^{-1}$ with respect to the galaxy rest frame. The same structure remains in the later NOT spectra, but it can be seen that the relative strengths of the features is changing. The final Keck spectrum spectacularly shows the three horns at $\sim-4240,-730$, and $+1400 \mathrm{~km} \mathrm{~s}^{-1}$. The evolution of the three components is particularly conspicuous between the P200 spectrum and the final Keck spectrum. We see that the emission line becomes more asymmetric with time, and that the blue horn is dominating the line profile at the last epoch, or rather that the red-most side of the line profile is suppressed. This is similar to the case of SN 1998S (Fig. 11). The lineprofile evolution of SNe 1993J and 1998S were discussed in some detail by Fransson et al. (2005). Several models for the geometry and dust distributions were tested, but none could 


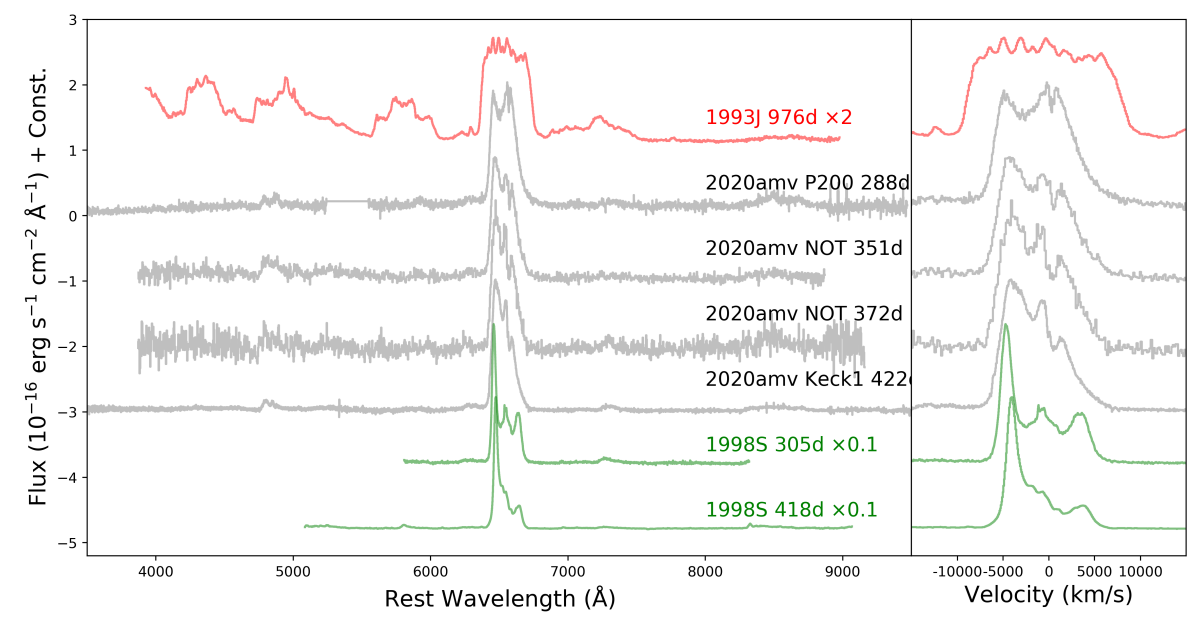

Fig. 11. Nebular spectra of SN 2020amv. Left: full range of the optical spectra, compared with SN 1993J (top, at 976 days, from Matheson et al. 2000) which also showed a boxy line profile, and with a sequence of spectra of SN 1998S (305 to 418 days, from Pozzo et al. 2004). Right: zoom-in on the $\mathrm{H} \alpha$ profile in velocity space. The profile is overall flat-topped and box-shaped in the first epoch, indicating emission from a thin dense shell formed when ejecta interact with the CSM. The later spectra show the development of an increasing overall asymmetry and structure, with a clear blueshifted horn at $-5000 \mathrm{~km} \mathrm{~s}^{-1}$. The evolution, with the suppression of the red side, is reminiscent of the evolution seen in SN $1998 \mathrm{~S}$.

convincingly explain the profiles of SN 1998S. The central component of the line profile would require an additional emission component - but the possibility that the central horn of the $\mathrm{H} \alpha$ line in SN 1998S was affected by host contamination was also mentioned (Fransson et al. 2005). In this regard, we note that the central component in the SN 2020amv Keck spectrum is real and clearly resolved at $900 \mathrm{~km} \mathrm{~s}^{-1}$. The host-galaxy $\mathrm{H} \alpha$ line from the same epoch has $F W H M \approx 240 \mathrm{~km} \mathrm{~s}^{-1}$. The offset between the two lines is $730 \mathrm{~km} \mathrm{~s}^{-1}$, with the central SN component blueshifted. Overall, the remarkable resemblance and the similar line-profile evolution between SN 1998S and SN 2020amv argue for a generic scenario rather than a fine-tuned geometry and dust distribution.

Also, finally looking at SN 2020jfv, the nebular emissionline spectrum is somewhat of an intermediate case between the two abovementioned SNe. This SN also caught our attention given the photometric behavior (rebrightening). Late-time SEDM spectra showed an unusual evolution - a strippedenvelope SN transforming into a SN II - which made us activate larger telescopes. The NOT data revealed a Balmer-dominated spectrum, but also clear [O I] $\lambda \lambda 6300,6364$ emission and what is likely [Ca II] at $7300 \AA$. The later Keck spectrum confirms this, and is even more dominated by $\mathrm{H} \alpha$ and $\mathrm{H} \beta$; we measured a flux ratio of $\mathrm{H} \alpha$ to [O I] $\lambda \lambda 6300,6364$ of $\sim 6.5$. The Ca II NIR triplet is also very weak. The peak of the $\mathrm{H} \alpha$ line profile is unfortunately damaged by a cosmic-ray hit in the high-S/N Keck spectrum. The final NOT spectrum, obtained after solar conjunction, shows basically only the Balmer lines.

The line profile of $\mathrm{H} \alpha$ in SN2020jfv has $F W H M \approx$ $2500 \mathrm{~km} \mathrm{~s}^{-1}$, which is virtually the same as for $\mathrm{H} \beta\left(2800 \mathrm{~km} \mathrm{~s}^{-1}\right.$, corrected for instrumental resolution; the host-galaxy lines are $750 \mathrm{~km} \mathrm{~s}^{-1}$ ). Whereas the host lines appear at $z=0.017$, both $\mathrm{H} \alpha$ and $\mathrm{H} \beta$ are blueshifted by $800 \mathrm{~km} \mathrm{~s}^{-1}$ from this. The extinctioncorrected Balmer ratio is $\sim 5$, indicating shock interaction. In Fig. 12 we compare two late spectra of SN 2020jfv with our best spectrum of SN 2020jfo, the P200 spectrum of SN 2020amv, and also with SN 2019oys which was a stripped-envelope SN that transformed into a SN IIn owing to late CSM interaction (Sollerman et al. 2020). We can see that the spectrum of SN 2020jfv is different from all of these comparison objects.
The right-hand panel of the figure zooms in on $\mathrm{H} \alpha$ in velocity space, and we note that the intermediate-width emission line of SN 2020jfv actually has structure on the red side of the line profile. This is significantly more subtle evidence of CSM interaction than for SN 2020amv.

\subsection{Uncertainties}

\subsubsection{Distance and extinction}

For the analysis and discussion so far we made use of the provided distances and assumed no additional extinction from the host galaxies of our SNe. In this section we discuss what the main uncertainties are provided the errors in the distance estimates, and discuss how the derived parameters would change if more extinction in the host galaxies would dim and redden the light from the SNe. These are the observational main caveats for most $\mathrm{SN}$ studies.

In Sect. 2 we declared that the distance to M61 is uncertain and adopted a distance modulus of $30.81 \pm 0.20 \mathrm{mag}$. This estimate comes from the Expanding Photosphere Method for a Type II SN (Bose \& Kumar 2014), and is also consistent with the peculiar-motion corrected luminosity-distance derived from standard cosmology and the observed redshift from NED. Such an uncertainty directly translates to a $20 \%$ error in the nickel mass estimates. There are, however, other estimates of the distance to M61 that are even larger (by almost 30\%, Pejcha \& Prieto 2015).

Extinction is sometimes even more difficult to determine. We have assumed no host galaxy extinction for the three SNe, mainly based on their blue colors. For SN 2020jfo there is some evidence for narrow $\mathrm{Na}$ I $\mathrm{D}$ lines in the Lick spectrum, where we can estimate an equivalent width of $\lesssim 0.7 \AA$ for the doublet. For example using Taubenberger et al. (2006) with $A_{V}=$ $3.1 \times 0.16 \times \mathrm{EW}(\mathrm{NaID})$ would give $0.3 \mathrm{mag}$ of extinction in the optical. In most regards such a $30 \%$ increase in flux is similar to adopting a larger distance, as discussed above.

With more color information available from the preexplosion HST imaging, such a reddening would also be of importance for the progenitor conclusions. However, as noted in 


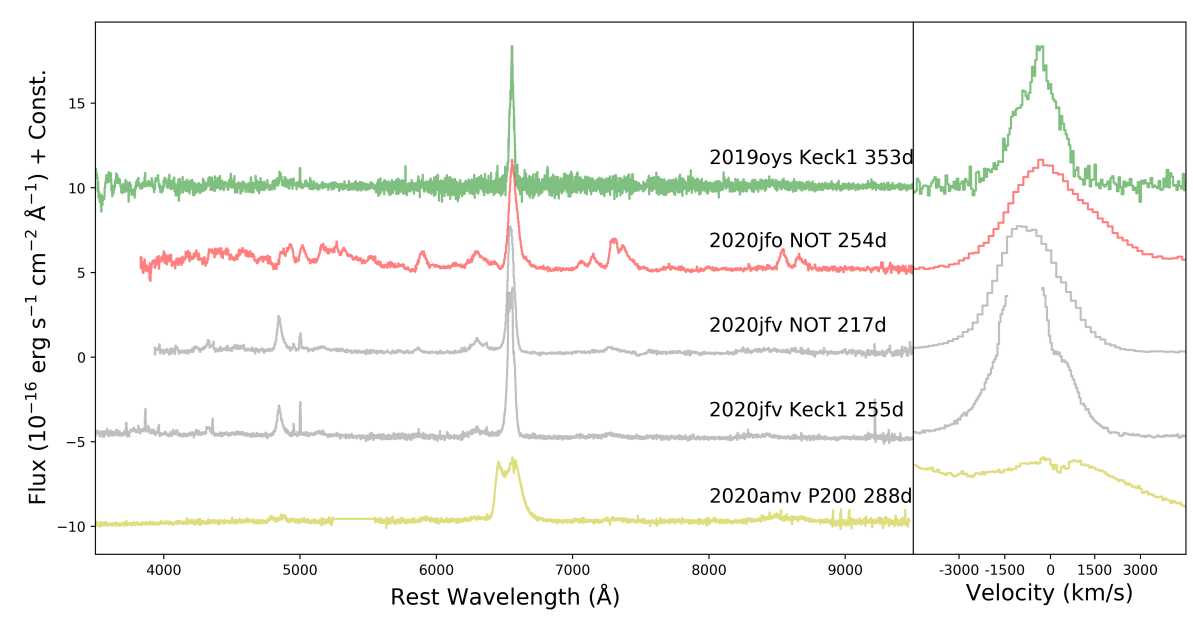

Fig. 12. Nebular spectra of SN 2020jfv compared with the normal SN 2020jfo, the CSM interacting SN 2020amv, and the transitional SN 2019oys (Sollerman et al. 2020). Right panel: zoom-in on the $\mathrm{H} \alpha$ line profile in velocity space. $\mathrm{H} \alpha$ dominates all these spectra, but the spectral evidence for CSM interaction is more subtle in SN 2020jfv, with a small structure visible at the red end of the line in the high-S/N Keck spectrum. The peak of this line is affected by a cosmic-ray hit and is therefore not shown.

Sect. 2.5 we detected the probable progenitor only in the reddest band, and it is fainter than expected by more than a magnitude. A host extinction correction of the magnitude suggested above would not resolve this issue.

A main effect from the uncertainty in distance and reddening is on the actual mass of radioactive nickel as mentioned above, the effects of both a larger distance and some host extinction could potentially increase the estimate from $0.025 M_{\odot}$ to $0.04 M_{\odot}$. Other parts of the analysis is less sensitive. For example the modeling of the nebular spectra relies on relative line luminosities for the ${ }^{58} \mathrm{Ni}$ analysis, which is virtually independent of both distance and extinction. Also the ZAMS mass estimate from the oxygen mass is derived by scaling the oxygen luminosity with the derived nickel mass, and both are similarly affected by the uncertainties.

Shortly, also for SNe 2020bmv and 2020jfo the discussion is similar as above, but there is not much analysis that is severely affected. The distance estimates are deduced from the redshifts, and at 74 and $200 \mathrm{Mpc}$ the effects of peculiar velocities is smaller $\left(V_{\mathrm{pec}}=150 \mathrm{~km} \mathrm{~s}^{-1}\right.$ gives at most $6 \%$ uncertainty in flux $)$, and is instead dominated by the everpresent uncertainty in the Hubble constant $\left( \pm 3 \mathrm{~km} \mathrm{~s}^{-1} \mathrm{Mpc}^{-1}\right.$ gives $9 \%$ in flux $)$. For these two peculiar SNe, the fact that the colors are not red is not much evidence against host galaxy reddening. None of our spectra show evidence for narrow ISM lines (SN 2020jfv have no constraining observations, whereas the NTT spectrum of SN 2020amv would reveal a $\mathrm{Na}$ ID of the same strengths as potentially present for SN 2020jfo). However, our discussion for these two SNe mostly concerned the line profiles and their evolution, and is not affected by these uncertainties.

\subsubsection{Methodology}

We can also briefly discuss the different methodologies used to infer the properties of SN 2020jfo and its progenitor. We have been able to use relatively well-established procedures to infer properties of the progenitor star from complementary routes such as pre-explosion progenitor imaging, bolometric light-curve modeling and nebular NLTE emission-line analysis. Whereas the derived oxygen mass implies a progenitor of ZAMS mass around $12 M_{\odot}$, the short LC plateau indicates a lower ejecta mass and the progenitor detection is fainter still.
This could imply interesting properties of the supernova, such as circumstellar dust around the progenitor destroyed in the explosion and extensive mass-loss affecting the hydrogen envelope, as mentioned in the next section. The tension could also indicate that some of the many assumptions inherent in these methods may not hold. More well-studied SNe where several lines of investigation can be applied are needed.

\section{Summary, interpretation, and conclusions}

In this paper we have presented the discovery, classification, and follow-up observations of the Type II SN 2020jfo, which exploded in the nearby spiral galaxy M61 in May 2020. We presented optical, NIR, and NUV photometry, as well as spectroscopy, for the first year of this transient, which was also followed by many other astronomers (both professional and amateur) around the globe. Even though the site was covered by PTF and ZTF for $11 \mathrm{yr}$ prior to explosion, we did not detect any pre-SN outbursts down to a magnitude of -11 .

The supernova has a well-constrained explosion epoch and rose to a maximum of $M_{r}^{\text {peak }}=-16.5$ mag in $\lesssim 5$ days. It was not detected at any significance in X-rays using Swift, but the UV LC was well covered. The plateau length of 65 days is on the short side of the SN IIP distribution. Using simple modeling we estimate an ejecta mass of $M_{\mathrm{ej}} \approx 5 M_{\odot}$, while the mass of radioactive nickel estimated from the late-time tail of the LC is $0.025 M_{\odot}$, although uncertainties in distance and host extinction could increase this by $\sim 50 \%$. The spectroscopic sequence of SN 2020jfo was largely obtained with robotic low-resolution spectrographs, but reveals normal SN II evolution dominated by Balmer lines with P Cygni profiles. This sort of sequence can routinely be achieved with SEDM-like spectrographs.

We also secured two epochs of spectropolarimetry. The S/N is rather low, but there is evidence of both continuum and line polarization. The interpretation is uncertain, but the modest continuum polarization may be due to an asymmetric distribution of radioactive elements, or of an asymmetric electron density structure. The higher line polarization suggests that $\mathrm{Ca}$ is not uniformly distributed within the ejecta and likely exists in clumps.

For the later nebular phases, we rely on ToO triggers on larger telescopes. The sequence of spectra of SN 2020jfo from 
the NOT displays slow evolution in the nebular phase. Lineflux measurements of the calibrated spectra indicate, when compared to detailed NLTE models, that the exploding progenitor had a ZAMS mass of $\sim 12 M_{\odot}$. Nebular emission line analysis also revealed a high abundance of stable nickel $\left({ }^{58} \mathrm{Ni}\right)$, with a mass ratio $M(\mathrm{Ni}) / M(\mathrm{Fe}) \approx 2$. Only a handful of CC SNe have previous estimates of this mass ratio, see Maeda et al. (2007), Jerkstrand et al. (2015c), Terreran et al. (2016), Tomasella et al. (2018), and Müller-Bravo et al. (2020).

The picture so far points to a value of around solar being most common - this corresponds to burning and ejection of oxygen-rich layers. SN 2020jfo joins a small group of SNe having an enhanced ratio of 2-4 times solar; others in this group are the normal Type IIP SN 2012ec (Jerkstrand et al. 2015c) and the broad-lined Type Ic SN 2006aj (Maeda et al. 2007). The result for SN 2020jfo adds another piece to the puzzle. With its moderate progenitor mass, there appears currently to be no simple dependency on progenitors mass, but rather that both lowmass and high-mass stars can achieve ejection of neutron-rich material.

Pre-explosion HST imaging reveal a putative source at the site of SN 2020jfo. The exact location was obtained with ground-based adaptive optics imaging, and confirmed with postexplosion HST imaging. The star is only detected in the reddest band with an absolute magnitude of $M_{F 814 W} \approx-5.8$, and nondetections in the bluer bands. This is fainter than expected and might imply circumstellar dust that was later destroyed by the SN explosion. The notably short plateau of SN 2020jfo implies that partial stripping of the envelope occurred at some episode before explosion, either through a stellar wind or mass exchange with a binary companion. Such mass loss would result in CSM, which could be consistent with the possible presence of circumstellar dust. If the CSM were set up by a vigorous pre-SN wind, it was not accompanied by any luminous outburst (Sect. 2.5).

We have examined BPASS binary models (Eldridge et al. 2019) for the mass range of $10-15 M_{\odot}$, as indicated for the ZAMS mass by the nebular line analysis. Although a number of models terminate near the observed $M_{F 814 W}$ (without the presence of further circumstellar extinction), the very low amount of $\mathrm{H}$ mass in these models at the time of explosion is more consistent with what we would expect for a SN IIb than a SN IIP progenitor. The reason for the faint, red possible detection of the SN 2020jfo progenitor, given the properties of the SN itself, therefore remains unknown and requires further study. Ultimately, the identification of the progenitor candidate must be confirmed by revisiting the site when the $\mathrm{SN}$ has sufficiently faded.

We have compared SN 2020jfo with two other ZTF SNe, all three being dominated by Balmer lines in the nebular spectra. SN 2020amv is also a SN II, but with a different LC and spectral evolution. The long-lived bumpy LC is a telltale sign of interaction with CSM. This is confirmed both by strong flashspectroscopy features at early times, and a broad, square-shaped nebular emission-line profile in $\mathrm{H} \alpha$, which furthermore consists of several components. The interpretation for such a line profile is emission from a cold dense shell developed by the reverse shock from interaction with dense CSM that the ejecta run into at later phases. CSM interaction is likely also the reason why SN 2020jfv rebrightens hundreds of days past explosion. This SN IIb initially did not have strong signatures of hydrogen, whereas the nebular spectrum is completely dominated by Balmer lines. The line shapes are somewhat intermediate between those discussed for the other two $\mathrm{SNe}$ above. Instead of evidence for CSM interaction in terms of a broad, flat-topped line profile, we see an intermediate-width asymmetric line, where optical-depth effects likely play a role in shaping the line. Interest in both of the latter targets was raised only after we realized their slowly evolving and rebrightening light curves, and in both cases initial SEDM data convinced us to also obtain spectra at larger telescopes. SEDM can thus operate both as a classification machine and as a science data provider (as for SN 2020jfo), but also as a way to investigate whether additional observations should be undertaken for any given transient. In SN 2020jfo itself, we see no evidence for CSM interaction, neither in the LC nor in the spectral evolution. However, based on the LC fit to the short plateau, and to the fast-declining late-time bolometric LC, we find evidence for a relatively low mass of hydrogen ejecta. Given the ZAMS mass derived from the nebular-line analysis, this points to a significant amount of mass loss. Since this CSM is not affecting the observed properties of the $\mathrm{SN}$, we suspect that this period of mass loss must have occurred at a substantially earlier phase of the progenitor's evolution.

Large surveys such as ZTF are now routinely discovering thousands of SNe. Part of the observations that were previously cumbersome and expensive to obtain, such as continuous LCs in several bands from early to late times and decent numbers of low-resolution spectra, are now virtually automatically available to the astronomical community - in particular, for nearby events such as SN 2020jfo. The challenge has moved to being able to digest and publish the available data, and to detect, select, and monitor events of particular interest. SNe 2020amv and 2020jfv were both unusually long lived with LCs that rebrightened. Dedicated spectroscopic follow-up observations were required to confirm CSM interaction as the powering mechanism for these objects.

Acknowledgements. We thank the staffs of the various observatories where data were obtained for their excellent assistance. Based on observations obtained with the Samuel Oschin Telescope 48-inch and the 60-inch Telescope at the Palomar Observatory as part of the Zwicky Transient Facility project. ZTF is supported by the US National Science Foundation (NSF) under grant AST-1440341 and a collaboration including Caltech, IPAC, the Weizmann Institute for Science, the Oskar Klein Center at Stockholm University, the University of Maryland, the University of Washington, Deutsches Elektronen-Synchrotron and Humboldt University, Los Alamos National Laboratories, the TANGO Consortium of Taiwan, the University of Wisconsin at Milwaukee, and Lawrence Berkeley National Laboratories. ZTF-II is supported by the National Science Foundation under Grant No. AST-2034437 and a collaboration including Caltech, IPAC, the Weizmann Institute for Science, the Oskar Klein Center at Stockholm University, the University of Maryland, Deutsches Elektronen-Synchrotron and Humboldt University, the TANGO Consortium of Taiwan, the University of Wisconsin at Milwaukee, Trinity College Dublin, Lawrence Livermore National Laboratories, and IN2P3, France. Operations are conducted by COO, IPAC, and UW. The SED Machine is based upon work supported by the NSF under grant AST1106171. The ZTF forced-photometry service was funded under the HeisingSimons Foundation grant \#12540303 (PI M. Graham). This work was supported by the GROWTH project funded by the NSF under PIRE grant 1545949 . The Oskar Klein Centre was funded by the Swedish Research Council. Gravitational Radiation and Electromagnetic Astrophysical Transients (GREAT) is funded by the Swedish Research council (VR) under Dnr 2016-06012. ECK is further supported by the Wenner-Gren Foundations. Much support to OKC involvement in ZTF was provided by the Knut and Alice Wallenberg foundation. Partially based on observations made with the Nordic Optical Telescope, operated by the Nordic Optical Telescope Scientific Association at the Observatorio del Roque de los Muchachos, La Palma, Spain, of the Instituto de Astrofisica de Canarias. Some of the data presented here were obtained with ALFOSC. Based on observations made with the NASA/ESA Hubble Space Telescope, obtained from the data archive at the Space Telescope Science Institute (STScI). STScI is operated by the Association of Universities for Research in Astronomy, Inc. under NASA contract NAS 5-26555. Support for program GO-16179 was provided by NASA through a grant from STScI. We thank T. de Jaeger, O. D. Fox, P. Kelly, N. Smith, S. Vasylyev, and W. Zheng for their assistance with this program. Some of the data presented herein were obtained at the W. M. Keck Observatory, which is 
operated as a scientific partnership among the California Institute of Technology, the University of California, and NASA; the observatory was made possible by the generous financial support of the W. M. Keck Foundation. The authors wish to recognize and acknowledge the very significant cultural role and reverence that the summit of Maunakea has always had within the indigenous Hawaiian community. We are most fortunate to have the opportunity to conduct observations from this mountain. A major upgrade of the Kast spectrograph on the Shane $3 \mathrm{~m}$ telescope at Lick Observatory was made possible through generous gifts from the Heising-Simons Foundation as well as William and Marina Kast. Research at Lick Observatory is partially supported by a generous gift from Google. Palomar Gattini-IR (PGIR) is generously funded by Caltech, the Australian National University, the Mt. Cuba Foundation, the Heising-Simons Foundation, and the Binational Science Foundation. PGIR is a collaborative project among Caltech, the Australian National University, University of New South Wales, Columbia University, and the Weizmann Institute of Science. Y.-L. K. has received funding from the European Research Council (ERC) under the European Unions Horizon 2020 research and innovation program (grant 759194 - USNAC). M. C. acknowledges support from the NSF through grant PHY-2010970. Funding for A. V. F.'s research group at U. C. Berkeley was provided by the Christopher R. Redlich Fund and the Miller Institute for Basic Research in Science (where A. V. F. is a Senior Miller Fellow). This work was partially funded by Kepler/K2 grant J1944/80NSSC19K0112, HST grant GO-15889 from STScI, and STFC grants ST/T000198/1 and ST/S006109/1. A. J. acknowledges funding from the European Research Council (ERC) through Starting Grant 803189. This work has made use of data from the Asteroid Terrestrial-impact Last Alert System (ATLAS) project. The Asteroid Terrestrial-impact Last Alert System (ATLAS project is primarily funded to search for near-Earth asteroids through NASA grants NN12AR55G, 80NSSC18K0284, and 80NSSC18K1575; by products of the asteroid search include images and catalogues from the survey area. The ATLAS science products have been made possible through the contributions of the University of Hawaii Institute for Astronomy, the Queen's University Belfast, STScI, the South African Astronomical Observatory, and The Millennium Institute of Astrophysics (MAS), Chile.

\section{References}

Ahn, C. P., Alexandroff, R., Allende Prieto, C., et al. 2014, ApJS, 211, 17 Anderson, J. P., González-Gaitán, S., Hamuy, M., et al. 2014, ApJ, 786, 67 Bellm, E. C., Kulkarni, S. R., Graham, M. J., et al. 2019, PASP, 131, 018002 Blagorodnova, N., Neill, J. D., Walters, R., et al. 2018, PASP, 130, 035003 Blanton, M. R., \& Roweis, S. 2007, AJ, 133, 734

Bose, S., \& Kumar, B. 2014, ApJ, 782, 98

Breeveld, A. A., Landsman, W., Holland, S. T., et al. 2011, in Gamma Ray Bursts 2010, eds. J. E. McEnery, J. L. Racusin, \& N. Gehrels, AIP Conf. Ser., 1358, 373

Bruch, R. J., Gal-Yam, A., Schulze, S., et al. 2021, ApJ, 912, 46

Burrows, D. N., Hill, J. E., Nousek, J. A., et al. 2005, Space Sci. Rev., 120, 165

Cardelli, J. A., Clayton, G. C., \& Mathis, J. S. 1989, ApJ, 345, 245

Cenko, S. B., Fox, D. B., Moon, D.-S., et al. 2006, PASP, 118, 1396

Chandra, P., Chevalier, R. A., Chugai, N., Milisavljevic, D., \& Fransson, C. 2020, ApJ, 902, 55

Chevalier, R. A., \& Fransson, C. 2017, in Thermal and Non-thermal Emission from Circumstellar Interaction, eds. A. W. Alsabti, \& P. Murdin, 875

Clocchiatti, A., \& Wheeler, J. C. 1997, ApJ, 491, 375

Dahiwale, A., \& Fremling, C. 2020a, Transient Name Server Classification Report, 2020-1491, 1

Dahiwale, A., \& Fremling, C. 2020b, Transient Name Server Classification Report, 2020-1948, 1

De, K., Hankins, M. J., Kasliwal, M. M., et al. 2020a, PASP, 132, 025001

De, K., Ashley, M. C. B., Andreoni, I., et al. 2020b, ApJ, 901, L7

Dekany, R., Smith, R. M., Riddle, R., et al. 2020, PASP, 132, 038001

Dolphin, A. 2016, Astrophysics Source Code Library [record ascl:1608.013

Eldridge, J. J., Guo, N. Y., Rodrigues, N., Stanway, E. R., \& Xiao, L. 2019, PASA, 36, e041

Evans, P. A., Beardmore, A. P., Page, K. L., et al. 2007, A\&A, 469, 379

Evans, P. A., Beardmore, A. P., Page, K. L., et al. 2009, MNRAS, 397, 1177

Faran, T., Poznanski, D., Filippenko, A. V., et al. 2014, MNRAS, 442, 844

Felipe Olivares, E., Hamuy, M., Giuliano, P., et al. 2010, ApJ, 715, 833

Filippenko, A. V. 1997, ARA\&A, 35, 309

Fransson, C., Challis, P. M., Chevalier, R. A., et al. 2005, ApJ, 622, 991

Fremling, C., Sollerman, J., Taddia, F., et al. 2016, A\&A, 593, A68

Fremling, C., Miller, A. A., Sharma, Y., et al. 2020, ApJ, 895, 32

Gal-Yam, A. 2017, in Observational and Physical Classification of Supernovae, eds. A. W. Alsabti, \& P. Murdin, 195
Gal-Yam, A., Arcavi, I., Ofek, E. O., et al. 2014, Nature, 509, 471

Gehrels, N., Chincarini, G., Giommi, P., et al. 2004, ApJ, 611, 1005

Graham, M. J., Kulkarni, S. R., Bellm, E. C., et al. 2019, PASP, 131, 078001

Hamuy, M., Pinto, P. A., Maza, J., et al. 2001, ApJ, 558, 615

Hiramatsu, D., Howell, D. A., Moriya, T. J., et al. 2021, ApJ, 913, 55

Irani, I., Zimmerman, E., Bruch, R., \& Yaron, O. 2020, Transient Name Server Classification Report, 2020-369, 1

Jäger, Z., Jr., Vinkó, J., Bíró, B. I., et al. 2020, MNRAS, 496, 3725

Jerkstrand, A. 2017, in Spectra of Supernovae in the Nebular Phase, eds. A. W. Alsabti, \& P. Murdin, 795

Jerkstrand, A., Fransson, C., Maguire, K., et al. 2012, A\&A, 546, A28

Jerkstrand, A., Ergon, M., Smartt, S. J., et al. 2015a, A\&A, 573, A12

Jerkstrand, A., Timmes, F. X., Magkotsios, G., et al. 2015b, ApJ, 807, 110

Jerkstrand, A., Smartt, S. J., Sollerman, J., et al. 2015c, MNRAS, 448, 2482

Jerkstrand, A., Ertl, T., Janka, H. T., et al. 2018, MNRAS, 475, 277

Kasliwal, M. M., Cannella, C., Bagdasaryan, A., et al. 2019, PASP, 131, 038003

Kawabata, M., Kawabata, K. S., Maeda, K., et al. 2018, PASJ, 70, 111

Larkin, J., Barczys, M., Krabbe, A., et al. 2006, in Society of Photo-Optical Instrumentation Engineers (SPIE) Conference Series, eds. I. S. McLean, \& M. Iye, 6269, 62691A

Li, H., \& McCray, R. 1993, ApJ, 405, 730

Lyman, J. D., Bersier, D., James, P. A., et al. 2016, MNRAS, 457, 328

Maeda, K., Kawabata, K., Tanaka, M., et al. 2007, ApJ, 658, L5

Masci, F. J., Laher, R. R., Rusholme, B., et al. 2019, PASP, 131, 018003

Matheson, T., Filippenko, A. V., Barth, A. J., et al. 2000, AJ, 120, 1487

Mauerhan, J. C., Filippenko, A. V., Zheng, W., et al. 2018, MNRAS, 478, 5050

Milisavljevic, D., Margutti, R., Kamble, A., et al. 2015, ApJ, 815, 120

Moore, A. M., \& Kasliwal, M. M. 2019, Nat. Astron., 3, 109

Müller-Bravo, T. E., Gutiérrez, C. P., Sullivan, M., et al. 2020, MNRAS, 497, 361

Nadyozhin, D. K. 2003, MNRAS, 346, 97

Nordin, J., Brinnel, V., Giomi, M., et al. 2020a, Transient Name Server Discovery Report, 2020-1248, 1

Nordin, J., Brinnel, V., Giomi, M., et al. 2020b, Transient Name Server Discovery Report, 2020-239, 1

Nyholm, A., Sollerman, J., Taddia, F., et al. 2017, A\&A, 605, A6

Nyholm, A., Sollerman, J., Tartaglia, L., et al. 2020, A\&A, 637, A73

Oke, J. B., \& Gunn, J. E. 1982, PASP, 94, 586

Oke, J. B., \& Gunn, J. E. 1983, ApJ, 266, 713

Oke, J. B., Cohen, J. G., Carr, M., et al. 1995, PASP, 107, 375

Pejcha, O., \& Prieto, J. L. 2015, ApJ, 799, 215

Perley, D. A. 2019, PASP, 131, 084503

Perley, D., Barbarino, C., Sollerman, J., et al. 2020a, Transient Name Server Classification Report, 2020-1259, 1

Perley, D., Barbarino, C., Sollerman, J., et al. 2020b, Transient Name Server AstroNote, 100,

Pozzo, M., Meikle, W. P. S., Fassia, A., et al. 2004, MNRAS, 352, 457

Prentice, S. J., Maguire, K., Boian, I., et al. 2020, MNRAS, 499, 1450

Prochaska, J., Hennawi, J., Westfall, K., et al. 2020, J. Open Sour. Softw., 5, 2308

Rigault, M., Neill, J. D., Blagorodnova, N., et al. 2019, A\&A, 627, A115

Roming, P. W. A., Kennedy, T. E., Mason, K. O., et al. 2005, Space Sci. Rev., 120,95

Serkowski, K., Mathewson, D. S., \& Ford, V. L. 1975, ApJ, 196, 261

Smartt, S. J., Valenti, S., Fraser, M., et al. 2015, A\&A, 579, A40

Smith, K. W., Smartt, S. J., Young, D. R., et al. 2020, PASP, 132, 085002

Sollerman, J., Cumming, R. J., \& Lundqvist, P. 1998, ApJ, 493, 933

Sollerman, J., Fransson, C., Barbarino, C., et al. 2020, A\&A, 643, A79

Spyromilio, J. 1994, MNRAS, 266, L61

Stanway, E. R., \& Eldridge, J. J. 2018, MNRAS, 479, 75

Strotjohann, N. L., Ofek, E. O., Gal-Yam, A., et al. 2021, ApJ, 907, 99

Tartaglia, L., Sollerman, J., Barbarino, C., et al. 2021, A\&A, 650, A174

Taubenberger, S., Pastorello, A., Mazzali, P. A., et al. 2006, MNRAS, 371, 1459

Terreran, G., Jerkstrand, A., Benetti, S., et al. 2016, MNRAS, 462, 137

Tomasella, L., Cappellaro, E., Pumo, M. L., et al. 2018, MNRAS, 475, 1937

Tonry, J. L., Denneau, L., Heinze, A. N., et al. 2018, PASP, 130, 064505

Tonry, J., Denneau, L., Heinze, A., et al. 2020, Transient Name Server Discovery Report, 2020-1243, 1

Valenti, S., Sand, D., Pastorello, A., et al. 2014, MNRAS, 438, L101

Wang, L., \& Wheeler, J. C. 2008, ARA\&A, 46, 433

Yang, S., Sollerman, J., Strotjohann, N. L., et al. 2021, A\&A, 655, A90

Yao, Y., Miller, A. A., Kulkarni, S. R., et al. 2019, ApJ, 886, 152

Yaron, O., \& Gal-Yam, A. 2012, PASP, 124, 668

Yaron, O., Perley, D. A., Gal-Yam, A., et al. 2017, Nat. Phys., 13, 510

Yuan, F., Jerkstrand, A., Valenti, S., et al. 2016, MNRAS, 461, 2003 